\title{
Conjugate Analysis of Rocket Nozzle Ablation
}

\author{
Peter G. Cross ${ }^{*}$ \\ Naval Air Warfare Center Weapons Division, China Lake, CA, 93555 \\ Iain D. Boyd ${ }^{\dagger}$ \\ University of Michigan, Ann Arbor, MI, 48109
}

\begin{abstract}
A methodology coupling the LeMANS flow solver to the MOPAR-MD material response solver is presented, enabling fully-coupled, conjugate, two-dimensional simulations of ablation of pyrolyzing materials in rocket nozzle applications. Five different treatments of the surface energy balance are presented, with increasing levels of fidelity. One of these methods eliminates transport coefficient assumptions and the need for pre-computed $B^{\prime}$ tables by directly using species diffusion at the ablating wall to compute char mass flux. Equilibrium surface chemistry is assumed; these calculations are performed using the MUTATION++ library. Ablation of the HIPPO nozzle test case is investigated using decoupled and conjugate analysis methods. A new baseline decoupled analysis is presented and compared to experimental data. Conjugate simulations are also performed using five different surface energy balance approaches and compared to the decoupled analysis results. The integrated equilibrium chemistry approach can fully capture the effects of ablation product species injection into the nozzle flowfield, in addition to the effects of recession, wall temperature, and blowing. By rigorously capturing the strong interactions and dependencies that exist between the reacting flowfield and the ablating material, improved analysis accuracy is anticipated.
\end{abstract}

\section{Nomenclature}

A Stoichiometric coefficient

$B^{\prime} \quad$ Nondimensional mass flux

$D \quad$ Diffusion coefficient, $\mathrm{m}^{2} / \mathrm{s}$

$d \quad$ Distance from cell centroid to wall, $\mathrm{m}$

$g_{H} \quad$ Enthalpy conductance, $\mathrm{kg} / \mathrm{m}^{2} \cdot \mathrm{s}$

$g_{M} \quad$ Mass transfer conductance, $\mathrm{kg} / \mathrm{m}^{2} \cdot \mathrm{s}$

$h \quad$ Specific enthlalpy, $\mathrm{J} / \mathrm{kg}$

$\tilde{J} \quad$ Element diffusion mass flux, $\mathrm{kg} / \mathrm{m}^{2} \cdot \mathrm{s}$

$\tilde{J}_{\text {error }}$ Diffusion correction term, $\mathrm{kg} / \mathrm{m}^{2} \cdot \mathrm{s}$

$\mathscr{M} \quad$ Molecular weight, $\mathrm{kg} / \mathrm{kmol}$

$\dot{m}^{\prime \prime} \quad$ Mass flux, $\mathrm{kg} / \mathrm{m}^{2} \cdot \mathrm{s}$

$N \quad$ Number of faces

$n \quad$ Flow solver iteration number

$P \quad$ Pressure, $\mathrm{Pa}$

$\dot{q}^{\prime \prime} \quad$ Heat flux, $\mathrm{W} / \mathrm{m}^{2}$

$T \quad$ Temperature, $\mathrm{K}$

$\mathrm{x} \quad$ Position vector, $\mathrm{m}$

$Y \quad$ Species mass fraction

$\tilde{Y} \quad$ Element mass fraction

*Aerospace Engineer, Aeromechanics \& Thermal Analysis Branch, 1900 N. Knox Road, M/S 6622, AIAA Senior Member.

$\dagger$ James E. Knott Professor, Department of Aerospace Engineering, 1320 Beale Ave., AIAA Fellow. 


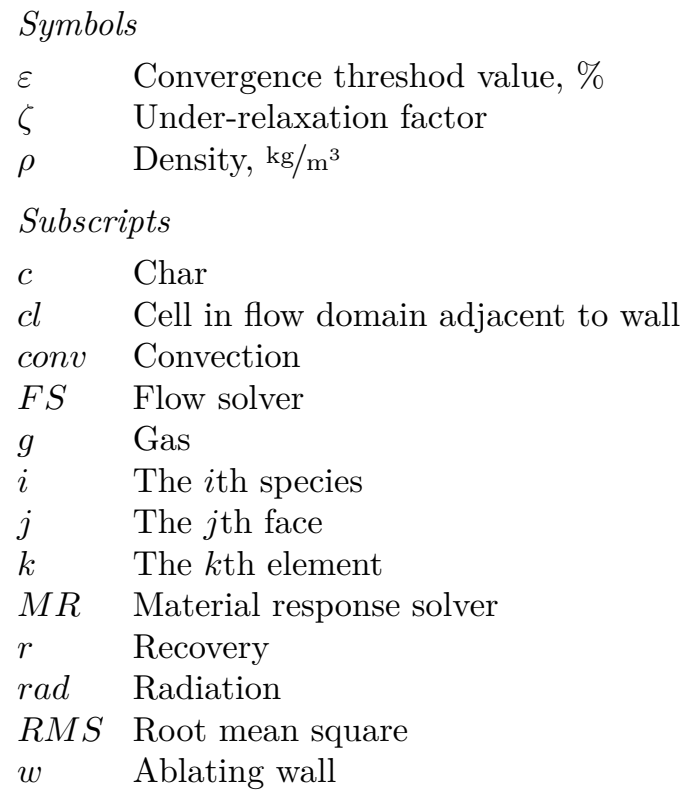

\section{Introduction}

Historically, ablation in rocket nozzles has been modeled using an approach in which the computation of the convective boundary conditions is decoupled from the thermal response of the ablator (see top diagram in Fig. 1). In this traditional approach, calculations to determine the convective heating environment in the nozzle are performed first. Often an empirical correlation called the Bartz equation ${ }^{1}$ is used to obtain the convective heating conditions. A common alternative is to use a $1 \mathrm{D}$ isentropic expansion calculation combined with integral boundary layer techniques, 2 but CFD techniques can also be used to determine the convective heating conditions. Usually these CFD analyses are performed assuming a nonablating wall. The thermal response and ablation of the thermal protection system (TPS) is then calculated with CMA 3 or a similar one-dimensional ablation code. To account for the effects of surface recession and blowing on the boundary layer, empirical corrections are applied by the material response solver to the pre-computed convective boundary conditions. The surface energy balance at the ablating surface is determined based upon transport coefficient assumptions and pre-computed $B^{\prime}$ tables.

With this decoupled approach it is not possible to rigorously account for geometric effects or thermochemical buffering of the boundary layer. Coupling between the flow solver and the material response solver is required in order to capture the effects of shape change due to recession.4 Also, char mass flux (recession rate) is sensitive to composition at the ablating surface,, 5 which is affected by the transport coefficient diffusion model assumption. Additionally, it has been found that the material response of the nozzle depends upon the wall temperature profile assumed when computing the cold wall heat flux. 6 Decoupled analyses are therefore unable to rigorously capture the mutual interactions that occur at the ablating boundary between the flowfield and the material response.

For conjugate analyses, the flowfield and material response are computed in a tightly-coupled manner (see lower diagram in Fig. 1), in order to capture the strong interactions and dependencies that exist between the reacting flowfield and the ablating material. As a result, improved analysis accuracy is anticipated.

Recent work at the University of Rome has investigated the effects of ablation on the flowfield within rocket nozzles. 11 Several of these studies modeled finite-rate surface chemistry, though some used an equilibrium chemistry approach that apparently did not make a transport coefficient assumption or use precomputed $B^{\prime}$ tables. However, this work assumed one-dimensional, steady-state ablation and did not consider the transient thermal response of the TPS material, nor did the simulations capture multidimensional effects, which can be important near the nozzle throat. Likewise, Thakre and Yang ${ }^{12}$ modeled nozzle flowfields assuming steady-state, one-dimensional ablation of non-charring materials.

Researchers at the University of Michigan 13 - 15 have performed coupled ablation analyses for external thermal protection systems (such as the IRV-2 vehicle and the Stardust reentry capsule) including the transient response of the TPS material. However, only non-charring materials have been modeled assuming 


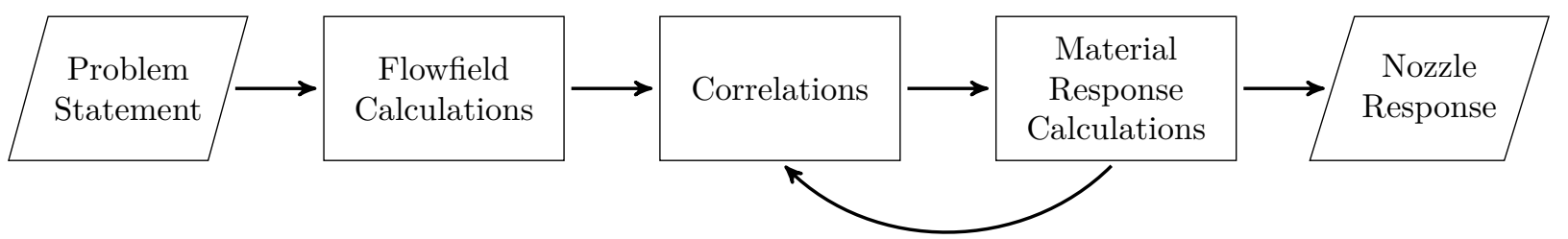

(a) Historical "decoupled" analysis methodology.

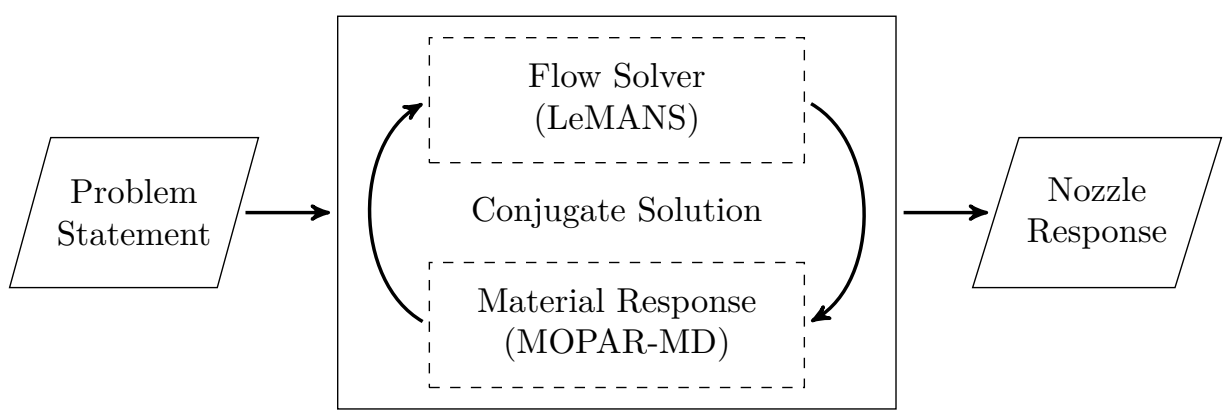

(b) Objective "conjugate" analysis methodology.

Figure 1. Flowcharts comparing the historical "decoupled" ablation analysis methodology and the "conjugate" ablation analysis methodology being pursued in this work.

two-dimensional heat transfer; conjugate simulations involving pyrolyzing materials have only been performed assuming one-dimensional heat transfer and ablation. Many of these studies used a surface energy balance approach based on $B^{\prime}$ tables, though some have explored finite-rate surface chemistry.

Kuntz et al. 1 investigated conjugate analysis of transient, two-dimensional ablation for the nosetip of the IRV-2 vehicle. However, only non-charring TPS materials were considered, using a surface energy balance approach that made the transport coefficient assumption and therefore required $B^{\prime}$ tables. The flow domain was periodically remeshed in order to capture the effects of surface recession. Researchers at NASA also investigated this test case, using a similar methodology. 16 This work assumed one-dimensional ablation.

Other research conducted at NASA investigated conjugate analyses of transient, one-dimensional ablation for the Stardust sample return capsule (which used a pyrolyzing TPS material); the surface energy balance was performed with the use of $B^{\prime}$ tables. .17 However, in this work the flow solver mesh was not updated to account for surface recession. More recent research conducted at NASA investigated ablation boundary conditions for flow solvers that directly model equilibrium surface chemistry and char mass flux based upon species diffusion at the wall; transport coefficient assumptions and $B^{\prime}$ tables were not required.5, 18 Ablation of pyrolyzing materials in external TPS applications was investigated; one-dimensional, steady-state ablation was assumed.

The goal of the present work is to establish and demonstrate a methodology enabling conjugate analyses of transient, two-dimensional ablation of pyrolyzing TPS materials (e.g. carbon-phenolic) in rocket nozzles. This paper begins with a brief description of the flow and material response solvers used in this work. The strategy used to couple these two codes together is then discussed, along with the criteria used to determine when the material response solver should be called to update the flow solver boundary conditions. Next, five different treatments of the surface energy balance are presented, including a detailed derivation for one technique that eliminates the need for transport coefficient assumptions and pre-computed $B^{\prime}$ tables. Finally, simulations of the HIPPO nozzle test case using these different conjugate analysis approaches are presented and compared to a baseline decoupled analysis.

\section{Methodology}

\section{II.A. LeMANS Flow Solver}

The flow solver used in this work is LeMANS, 19,20 a multi-species, reacting Navier-Stokes solver developed at the University of Michigan. This code was originally created for the purpose of studying thermal and chemical nonequilibrium phenomena that occur in two- or three-dimensional, laminar, hypersonic flowfields, 
but additional features have recently been added to the code enabling rocket nozzle flowfields to be modeled. 6 Turbulence can be handled using the Menter BSL and SST k- $\omega$ turbulence models. Gas-particle mixtures (caused by the presence of alumina particles formed during the combustion of the aluminum in the solid propellant) are modeled using an equilibrium "two gas" method that treats the condensed phase as an additional gas species with special properties. Multiple temperatures are used to model the different energy modes of all species, and different transport property models are available. A modified Steger-Warming Flux Vector Splitting Scheme is used for computing the inviscid fluxes, while a central-difference scheme is used for the viscous fluxes; LeMANS is second-order accurate in space. Steady-state solutions are obtained through a time-marching method. Integration is generally performed using a line-implicit scheme, though a point-implicit scheme is also available. Parallelization is achieved by using MPI and METIS libraries.

\section{II.B. MOPAR-MD Material Response Solver}

The material response solver used in this work, MOPAR-MD, 6, 21 was originally developed at the University of Michigan to explore the ablation response of non-charring materials with anisotropic thermal conductivity. With recent enhancements, it is now possible to also model the ablation of pyrolyzing materials; an unlimited number of decomposition components are permitted. Transport of the pyrolysis gases through the porous char is modeled using a form of Darcy's law that can accommodate anisotropic permeability. The ablation boundary condition follows a typical method based on $B^{\prime}$ tables, and makes the unity Lewis number assumption (meaning that the mass and energy transport coefficients are equal). MOPAR-MD can model two-dimensional or axisymmetric geometries with unstructured meshes, and deforms the mesh to account for surface recession. Time integration is performed using a first-order implicit scheme; spatial discretization is performed using a second-order control volume finite element method. The mesh deformation, energy, solid phase continuity, and gas phase continuity equations are loosely-coupled and solved sequentially in an iterative process each time step. MOPAR-MD is a serial code, which places limitations on the size of a simulation that can be affordably completed.

\section{II.C. Coupling Strategy}

In this work the LeMANS flow solver is coupled to the MOPAR-MD material response solver, enabling fully-coupled, conjugate simulations of ablation of pyrolyzing materials. The pressure trace for a rocket motor firing is divided into a number of discrete time points (see Fig. 2). At each time point a steady-state flowfield solution is obtained using the flow solver. The wall boundary conditions required by the flow solver are obtained from the material response solver, which is linked into the flow solver executable as a boundary condition routine. At each time point, the material response solver performs a transient analysis starting

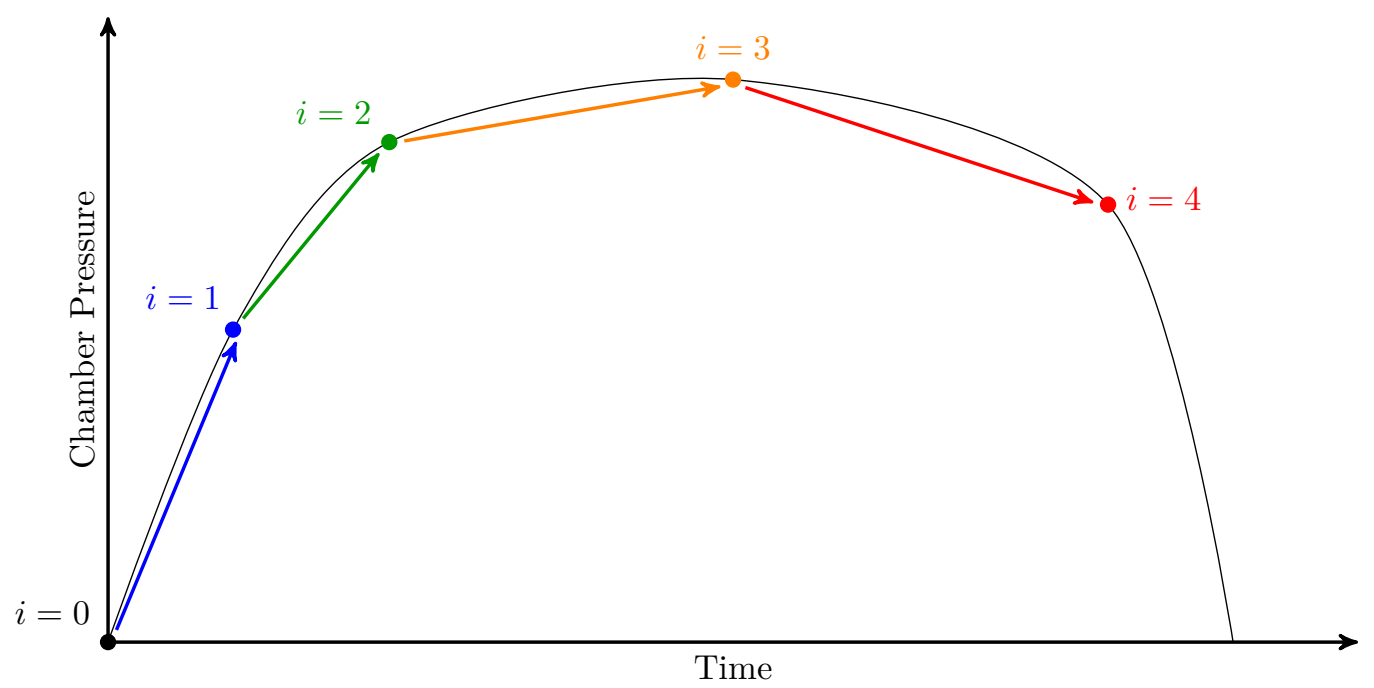

Figure 2. Diagram illustrating how a pressure trace is divided into a number of discrete time points (solid circles) at which steady-state flowfield solutions are obtained. Transient material response calculations (represented by arrows) are performed to obtain the flowfield boundary conditions for each time point. 
Table 1. Variables exchanged between the flow solver and the material response solver for multiple approaches to treating the surface energy balance at the ablating wall.

\begin{tabular}{ccc}
\hline Methodology & Flow $\rightarrow$ Solid & Solid $\rightarrow$ Flow \\
\hline Noncatalytic Wall (NCW) & $P, \dot{q}^{\prime \prime}, h_{r}$ & $T, \dot{m}^{\prime \prime}, \mathbf{x}$ \\
Noncatalytic Wall Using Coefficients (NCWC) & $P, g_{H}, h_{r}$ & $T, \dot{m}^{\prime \prime}, \mathbf{x}$ \\
Ablating Wall Using Tables (AWT) & $P, \dot{q}^{\prime \prime}, h_{r}$ & $T, \dot{m}^{\prime \prime}, \mathbf{x}, Y_{i}$ \\
Ablating Wall Using Tables and Coefficients (AWTC) & $P, g_{H}, h_{r}$ & $T, \dot{m}^{\prime \prime}, \mathbf{x}, Y_{i}$ \\
Integrated Equilibrium Chemistry (IEC) & $P, \dot{q}^{\prime \prime}, \dot{m}_{c}^{\prime \prime}, h_{w}$ & $T, \dot{m}_{g}^{\prime \prime}, \mathbf{x}$ \\
\hline
\end{tabular}

from the solution obtained at the previous time point, using as boundary conditions values passed from the flow solver. These values are linearly interpolated between time points. The variables passed between solvers depend on the surface energy balance approach (see section II.E), as given in Table 1 .

An under-relaxation factor is applied to the variables passed from the material response solver to the flow solver:

$$
\theta=(1-\zeta) \theta_{F S}+\zeta \theta_{M R}
$$

Here $\theta$ represents any variable of interest. Thus, the new values for these flowfield boundary variables are a blend of the previously-used values and the latest values predicted by the material response solver. As will be shown later (see section III.C), this under-relaxation factor helps with the convergence of the conjugate solution. Generally, the under-relaxation factor is applied to the temperature, mass flux, wall position, and species mass fractions. No under-relaxation factor is applied to pyrolysis gas mass flux when using the integrated equilibrium chemistry method. It should also be noted that the flowfield and material response meshes do not need to have coincident nodes; interpolation is used to map values between meshes. This adds flexibility and allows each mesh to be optimized independently.

A fully converged conjugate solution is obtained for each time point before advancing to the next. This requires an iterative process with the material response solver being called multiple times for each time point, as illustrated in Fig. 3. Starting with the solution from the previous time point, a flow solver iteration is performed, producing updated values for the material response boundary conditions (in this example, pressure, heat flux, and recovery enthalpy). At the end of every flow iteration, a check is performed to determine whether or not the material response solver should be called to update the ablating wall boundary conditions (see section II.D). If it is determined that the wall values should be updated, the material response solver is called, which performs a transient thermal analysis starting from the previous time point. This yields updated values for the flow solver boundary conditions (e.g. wall temperature, injected mass flux, and wall position). The flow solver then updates the flow domain mesh to account for wall recession, and a new

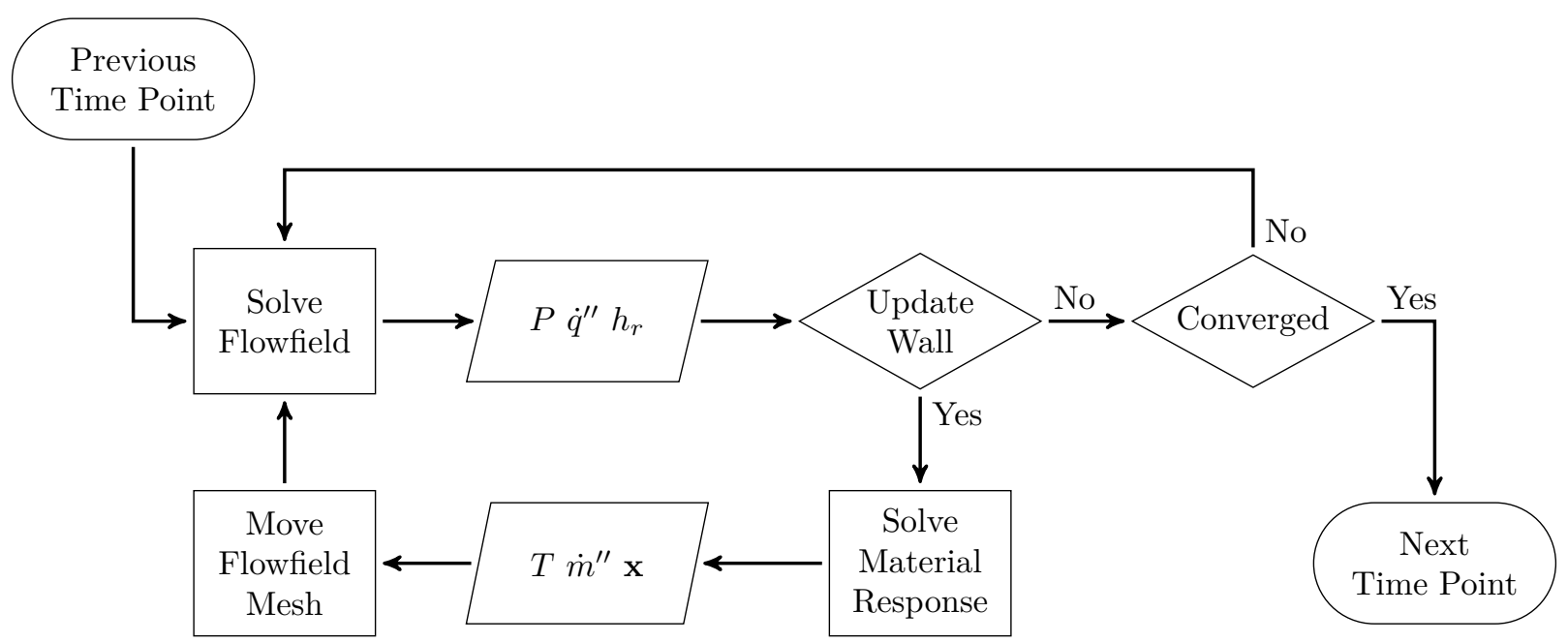

Figure 3. Flowchart illustrating the approach taken to obtain a converged conjugate solution for a given time point. 
flow iteration is performed. If the first check determines that it is not necessary to update the wall values, a second check is performed to determine if convergence has been achieved. If convergence is not achieved, the process continues with another flowfield iteration, otherwise, the conjugate solution can advance to the next time point.

\section{II.D. Surface Update Criteria}

In this effort, the material response solver is called in order to update the flowfield wall boundary conditions once three different criteria are all satisfied. The first criterion:

$$
n-n_{M R} \geq n_{\min }
$$

requires that some minimum number of flow iterations be completed between calls to the material response solver. This helps ensure that the flow has sufficient time to respond to the latest wall conditions before updating the wall again. The second criterion:

$$
\max \left(100\left|\frac{\dot{q}_{j}^{\prime \prime n}-\dot{q}_{j}^{\prime \prime n-1}}{\dot{q}_{j}^{\prime \prime n-1}}\right|\right)<\varepsilon_{\dot{q}^{\prime \prime}}
$$

looks at the maximum change in heat flux between two subsequent flow iterations, and is a measure of the convergence of the flowfield. This criterion prevents the wall values from being updated while the flowfield is still undergoing significant changes. The third and final criterion:

$$
\begin{gathered}
100\left|\frac{\dot{q}_{R M S}^{\prime \prime n}-\dot{q}_{R M S}^{\prime \prime n_{M R}}}{\dot{q}_{R M S}^{\prime \prime n}}\right| \geq \varepsilon_{\dot{q}_{R M S}^{\prime \prime}} \\
\dot{q}_{R M S}^{\prime \prime}=\sqrt{\frac{1}{N} \sum_{j}^{N} \dot{q}_{j}^{\prime 2}}
\end{gathered}
$$

looks at the change in the root mean square (RMS) heat flux since the last time the material response solver was called, and is a measure of how much the flowfield has changed since the last time the wall values were updated. This criterion prevents the material response solver from being needlessly called if the wall heat flux has not changed significantly.

The three parameters on the right hand side of Eqs. (2) through (14) are threshold values that must be specified by the user; trial-and-error is required to identify appropriate values for a given problem. The following values are found to be generally suitable for this effort, and are used for most simulations presented in this paper:

$$
\begin{gathered}
n_{\text {min }}=2000 \\
\varepsilon_{\dot{q}^{\prime \prime}}=0.01 \% \\
\varepsilon_{\dot{q}_{R M S}^{\prime \prime}}=0.05 \%
\end{gathered}
$$

\section{II.E. Surface Energy Balance}

Five different approaches for treating the surface energy balance are identified and implemented into the conjugate ablation analysis code.

\section{II.E.1. Noncatalytic Wall (NCW)}

In the NCW coupling approach, a noncatalytic wall (zero species mass fraction gradient in the wall-normal direction) boundary condition is used in the flow solver, and the raw heat flux is passed to the material response solver. This is the same surface energy balance approach used by other researchers at the University of Michigan for some conjugate ablation studies for external thermal protection system applications. 13,15 The surface energy balance is performed in the material response solver using pre-computed $B^{\prime}$ tables. Recall that the net heat flux to an ablating surface is given by: 6

$$
\begin{gathered}
\dot{q}_{\text {net }}^{\prime \prime}=\underbrace{\dot{q}_{c o n v}^{\prime \prime}-\dot{q}_{g}^{\prime \prime}-\dot{q}_{c}^{\prime \prime}}_{\text {ablation }}+\dot{q}_{r a d_{i n}}^{\prime \prime}-\dot{q}_{r a d_{o u t}}^{\prime \prime} \\
6 \text { of } 22
\end{gathered}
$$


In this approach, the heat flux obtained from the flow solver is used directly as the convective heat flux; no approximation or modification is used:

$$
\dot{q}_{\text {conv }}^{\prime \prime}=\dot{q}_{F S}^{\prime \prime}
$$

However, the heat fluxes due to pyrolysis gas and char mass flux require values for the ablating wall enthalpy $h_{w}$ and the nondimensional char mass flux $B_{c}^{\prime}$ for closure:

$$
\begin{gathered}
\dot{q}_{g}^{\prime \prime}=\dot{m}_{g}^{\prime \prime} h_{w} \\
\dot{q}_{c}^{\prime \prime}=\dot{m}_{c}^{\prime \prime} h_{w}=B_{c}^{\prime} g_{H} h_{w}
\end{gathered}
$$

These values are tabulated in the $B^{\prime}$ tables as functions of wall temperature, pressure, and nondimensional pyrolysis gas mass flux:

$$
\begin{aligned}
h_{w} & =f\left(T_{w}, P, B_{g}^{\prime}\right) \\
B_{c}^{\prime} & =f\left(T_{w}, P, B_{g}^{\prime}\right)
\end{aligned}
$$

Pyrolysis gas mass flux is nondimensionalized by an enthalpy conductance; note that the unity Lewis number assumption (mass transfer coefficient and energy transfer coefficient are the same, $g_{M}=g_{H}$ ) is made:

$$
B_{g}^{\prime}=\frac{\dot{m}_{g}^{\prime \prime}}{g_{M}}=\frac{\dot{m}_{g}^{\prime \prime}}{g_{H}}
$$

Thus, to use pre-computed $B^{\prime}$ tables to provide closure for the net heat flux to the ablating boundary, it is necessary to compute an enthalpy conductance (enthalpy-based convection coefficient):

$$
g_{H}=\frac{\dot{q}_{F S}^{\prime \prime}}{h_{r}-h_{w}}
$$

In this calculation the recovery enthalpy $h_{r}$ is assumed to be equal to the gas-phase stagnation enthalpy. Since wall enthalpy $h_{\mu}$ is obtained from the $B^{\prime}$ tables and is therefore a function of $g_{H}$, an iterative procedure is used to solve Eq. (13).

\section{II.E.2. Noncatalytic Wall Using Coefficients (NCWC)}

In this approach, a noncatalytic wall boundary condition is still used in the flow solver, but an enthalpy conductance (heat transfer coefficient) is passed to the material response solver instead of the raw heat flux. The enthalpy conductance is computed within the flow solver according to:

$$
g_{H}=\frac{\dot{q}_{F S}^{\prime \prime}}{h_{r}-h_{F S}}
$$

where $h_{F S}$ is the enthalpy of the gas-phase species present at the noncatalytic wall. Note that the difference between the enthalpy conductance computed by Eq. (14) for the NCWC method and the enthalpy conductance computed by Eq. (13) for closure of the NCW method is the choice of wall enthalpy. For the NCW method the ablating wall enthalpy from the $B^{\prime}$ tables is used, while for the NCWC method the noncatalytic wall enthalpy from the flow solver is used, which is more appropriate.

Within the material response solver, this enthalpy conductance is used to compute the convective heat flux:

$$
\dot{q}_{\text {conv }}^{\prime \prime}=g_{H}\left(h_{r}-h_{w}\right)
$$

where the wall enthalpy $h_{w}$ comes from the $B^{\prime}$ tables. Computation of the heat fluxes due to pyrolysis gas and char mass flux is the same as for the NCW method.

Since the wall enthalpy for a noncatalytic wall flow solution will in general be different than the ablating wall enthalpy (due to different compositions at the wall), then the heat flux used by the material response solver will not match the heat flux computed by the flow solver. However, this is consistent with the traditional decoupled ablation analysis approach, and actually represents an improvement over the decoupled approach, since the effects of wall temperature and mass injection are being accounted for directly in the computation of the enthalpy conductance (instead of being approximated with correlations). Additionally, using a heat transfer coefficient is a "softer" boundary condition than using a raw flux. It is therefore expected that simulations using this approach will be more robust and stable, and it should be possible to employ larger spacing between time points.

$$
7 \text { of } 22
$$




\section{II.E.3. Ablating Wall Using Tables (AWT)}

The AWT method is similar to the NCW method in that a raw heat flux is passed from the flow solver to the material response solver. However, a noncatalytic wall boundary condition is not used. Instead, the species mass fraction at the wall is specified according to the equilibrium composition at the ablating wall, which is obtained as part of the procedure used to compute the $B^{\prime}$ tables. Normally this composition data is not retained, but for the AWT method the species mass fractions are stored in an "extended" $B^{\prime}$ table as functions of wall temperature, pressure, and nondimensional pyrolysis gas mass flux:

$$
Y_{i}=f\left(T_{w}, P, B_{g}^{\prime}\right)
$$

The material response solver interpolates from this "extended" $B^{\prime}$ table and passes the resultant species mass fractions at the ablating wall back to the flow solver. This surface energy balance approach is similar to that used by Olynick et al.17

Species mass fractions at a given point in the flow can change by several orders of magnitude between two different time points. Such a situation can arise, for example, when the temperature at a given location at one time point is low enough so that ablation does not occur, but at the next time point has increased to the point where ablation does occur. In this situation, applying an under-relaxation factor to the species mass fractions is unable to adequately capture this significant change in composition. It is found that applying the under-relaxation factor to the logarithm of the species mass fractions provides a much better adjustment of the wall composition.

\section{II.E.4. Ablating Wall Using Tables and Coefficients (AWTC)}

This method is similar to the AWT method, except that an enthalpy conductance is passed from the flow solver to the material response solver, instead of the raw heat flux. In this regard it is similar to the NCWC method. Once a converged coupled solution is obtained, the wall enthalpy computed by the flow solver should be the same as the ablating wall enthalpy from the $B^{\prime}$ tables. Therefore, the heat flux computed by the flow solver will match the heat flux used in the material response solver. However, this will not necessarily be the case as the solution is developing (mainly due to the under-relaxation factor applied to the wall mass fractions). This approach is very similar to that used by Kuntz et al. 1 t

\section{II.E.5. Integrated Equilibrium Chemistry (IEC)}

The preceding four methods all use $B^{\prime}$ tables within the material response solver to perform the surface energy balance. In the IEC approach, $B^{\prime}$ tables are completely abandoned. Instead, the equilibrium chemistry calculations at the surface are integrated into the LeMANS flow solver. Equilibrium chemistry ablation is computed based on the diffusive fluxes at the wall and the injection of the pyrolysis gases without resorting to a transport coefficient assumption. This approach is similar to that presented by Johnston et al. $.5,18$

Consider a thin control volume located just above an ablating surface, as illustrated in Fig. 4 . Assuming no mechanical removal of char, element conservation and mass conservation dictate that:22, 23.

$$
\tilde{J}_{k_{w}}+\left(\dot{m}_{g}^{\prime \prime}+\dot{m}_{c}^{\prime \prime}\right) \tilde{Y}_{k_{w}}=\dot{m}_{g}^{\prime \prime} \tilde{Y}_{k_{g}}+\dot{m}_{c}^{\prime \prime} \tilde{Y}_{k_{c}}
$$

The first term on the left hand side is the diffusion of element $k$ away from the wall into the flow. Normally this diffusion is approximated using a transport coefficient, leading to the formulation of the $B^{\prime}$ tables. In

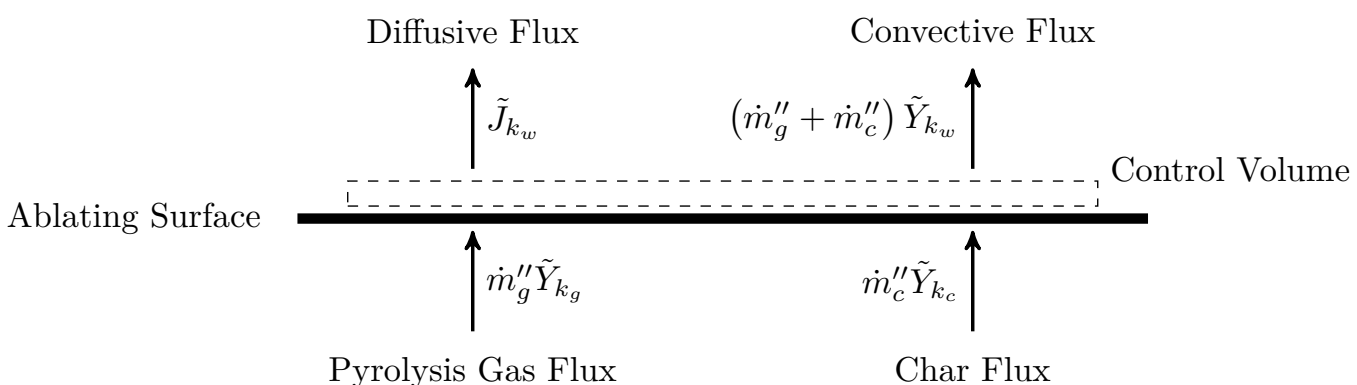

Figure 4. Diagram illustrating elemental mass balance at an ablating surface. 
the IEC approach, however, this diffusive flux is computed using the modified Fick's model employed by the LeMANS flow solver. Assuming that the diffusive flux is dominated by the gradient in the wall-normal direction, this results in:

$$
\begin{gathered}
\tilde{J}_{k_{w}}=\frac{\rho D}{d}\left(\tilde{Y}_{k_{w}}-\tilde{Y}_{k_{c l}}\right)-\tilde{Y}_{k_{w}} \tilde{J}_{\text {error }} \\
\tilde{J}_{\text {error }}=\sum_{k} \frac{\rho D}{d}\left(\tilde{Y}_{k_{w}}-\tilde{Y}_{k_{c l}}\right)
\end{gathered}
$$

$\tilde{J}_{\text {error }}$ is a correction term to ensure that the net mass diffusion is zero. Substituting Eq. (18) into Eq. (17) and solving for the elemental mass fraction at the wall (assuming that $\tilde{J}_{\text {error }}$ is insensitive to $\tilde{Y}_{k_{w}}$ ) yields:

$$
\tilde{Y}_{k_{w}}=\frac{\dot{m}_{g}^{\prime \prime} \tilde{Y}_{k_{g}}+\dot{m}_{c}^{\prime \prime} \tilde{Y}_{k_{c}}+\frac{\rho D}{d} \tilde{Y}_{k_{c l} l}}{\dot{m}_{g}^{\prime \prime}+\dot{m}_{c}^{\prime \prime}+\frac{\rho D}{d}-\tilde{J}_{\text {error }}}
$$

which is the desired expression giving the elemental composition at the ablating wall. However, the char mass flux remains an unknown.

Ablation occurs when $\dot{m}_{c}^{\prime \prime}$ is sufficient to saturate the equilibrium composition adjacent to the wall with the char species (generally assumed to be carbon), i.e. $Y_{c}=0$. Larger values of $\dot{m}_{c}^{\prime \prime}$ will super-saturate the mixture, resulting in $Y_{c}>0$, but leaving the gas-phase composition unchanged. Thus, the gas-phase composition at the ablating wall can be determined by setting $\dot{m}_{c}^{\prime \prime}$ to a large value that will ensure supersaturation of the equilibrium solution. In this work the char mass flux used in Eq. (20) is computed as:

$$
\dot{m}_{c}^{\prime \prime}=\max \left(100 \dot{m}_{g}^{\prime \prime}, 200 \mathrm{~kg} / \mathrm{m}^{2} \mathrm{~s}\right)
$$

where the constants have been arbitrarily selected and could potentially be reduced.

Once the elemental mass fractions at the super-saturated wall have been determined, the Mutation++ library 24 is used to obtain the corresponding equilibrium solution. The saturated equilibrium composition is then obtained by setting $Y_{c}=0$ and re-normalizing the gas-phase mass fractions. Solving Eq. (20) for the char mass flux gives:

$$
\dot{m}_{c}^{\prime \prime}=\frac{\dot{m}_{g}^{\prime \prime}\left(\tilde{Y}_{k_{g}}-\tilde{Y}_{k_{w}}\right)-\frac{\rho D}{d}\left(\tilde{Y}_{k_{w}}-\tilde{Y}_{k_{c l}}\right)+\tilde{J}_{\text {error }} \tilde{Y}_{k_{w}}}{\tilde{Y}_{k_{w}}-\tilde{Y}_{k_{c}}}
$$

where it is only necessary to use the mass fraction of a single element at the saturated wall. This method of determining $\dot{m}_{c}^{\prime \prime}$ is different from that used by Johnston et al.,5, 18 who instead relied on a curve fit for an equilibrium relation between gas-phase and condensed-phase carbon.

The elemental mass fractions required in these calculation can be obtained from the species mass fractions according to:

$$
\tilde{Y}_{k}=\mathscr{M}_{k} \sum_{i} A_{k i} \frac{Y_{i}}{\mathscr{M}_{i}}
$$

where $A_{k i}$ is the stoichiometric coefficient giving the number of atoms of element $k$ present in one molecule of species $i$.

When calling the material response solver, the convective heat flux, char mass flux, and wall enthalpy are passed from the flow solver to the material response solver, and are assumed to vary linearly between time points. Excluding radiation (which is easily handled separately), the net heat flux to the ablating surface is computed in the material response solver as:

$$
\dot{q}_{n e t}^{\prime \prime}=\dot{q}_{F S}^{\prime \prime}-\dot{m}_{g}^{\prime \prime} h_{w}-\dot{m}_{c}^{\prime \prime} h_{w}
$$

This approach allows the pyrolysis gas mass flux (and associated heat flux) to vary in a nonlinear way between time points (while the other terms are linear).

\section{II.E.6. Methodology Comparison}

As is shown in section (III.D), the NCW method significantly over-predicts the ablation response of the HIPPO nozzle. This is mainly because the noncatalytic wall boundary condition used in the flow solver over-predicts the heat flux to the wall of the nozzle. This is illustrated in Fig. 5, where the convection

$$
9 \text { of } 22
$$




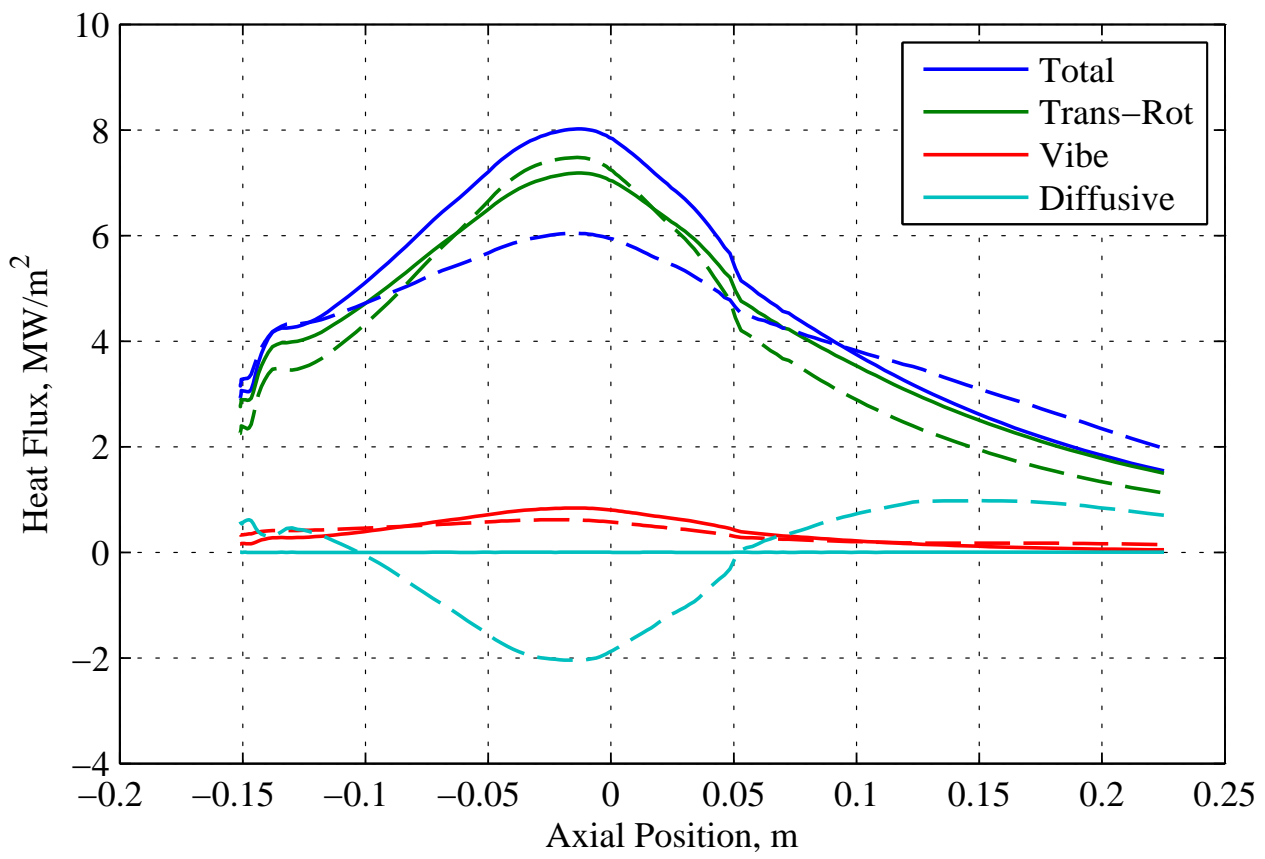

Figure 5. Convection heat flux components as a function of axial position for the HIPPO nozzle at $t=0.2 \mathrm{~s}$, as computed with the NCW method (solid lines) and the AWT method (dashed lines).

heat flux components as computed with the NCW and AWT methods are compared for $t=0.2 \mathrm{~s}$. The heat flux due to the translation-rotational and vibrational temperature gradients are similar for the two methods. However, for the AWT method the heat flux component associated with species diffusion is negative near the throat (where ablation is occurring) and positive near the nozzle exit (where ablation is not occurring). Since (by definition) the NCW method does not include this negative contribution from the diffusive heat flux, the total heat flux is over-predicted.

The noncatalytic wall boundary condition causes the species composition at the wall to be significantly different from the actual ablating wall composition. As a consequence, for the HIPPO nozzle the ablating wall enthalpy is greater than the noncatalytic wall enthalpy (see Fig. 6). Recall Eq. (15) suggests that heat flux should decrease as wall enthalpy increases. It is therefore reasonable to expect that the heat flux to the ablating wall should actually be less than that predicted by the noncatalytic wall. For ablation in air the noncatalytic wall boundary condition is considered to provide the lower bound on heat flux, 13 but for ablation in rocket nozzles the noncatalytic wall boundary condition appears to provide the upper bound on heat flux.

A further contributing factor to the over-prediction of the ablation response by the NCW approach is the method used to compute the enthalpy conductance, which is required in order to use the $B^{\prime}$ tables for closure of the surface energy balance. Recall for the NCW method that enthalpy conductance is computed based upon the ablating wall enthalpy (Eq. (13)). Therefore, a much larger value for enthalpy conductance is obtained than if the noncatalytic wall enthalpy was used. This is illustrated in Fig. 7, where enthalpy conductance is computed based on the total heat flux for the NCW method (shown in Fig. 5) and the two different wall enthalpies shown in Fig. 6. Since char mass flux (and therefore recession rate) is proportional to enthalpy conductance, greater surface recession will be predicted when the noncatalytic heat flux is converted to an enthalpy conductance using the ablating wall enthalpy. Since the purpose of enthalpy conductance is to characterize a convective heating environment, enthalpy conductance should therefore always be computed using the same wall enthalpy as was used in the computation of the convection heat flux (as is the case for the other methods considered). Because of these shortcomings, the NCW method is found to be unsuitable for modeling ablation in rocket nozzles.

The NCWC method is most similar to the decoupled analysis technique in that a noncatalytic wall boundary condition is used in the flow solver to obtain an enthalpy conductance, which is applied as a boundary condition in the material response solver. The NCWC method improves upon the decoupled 


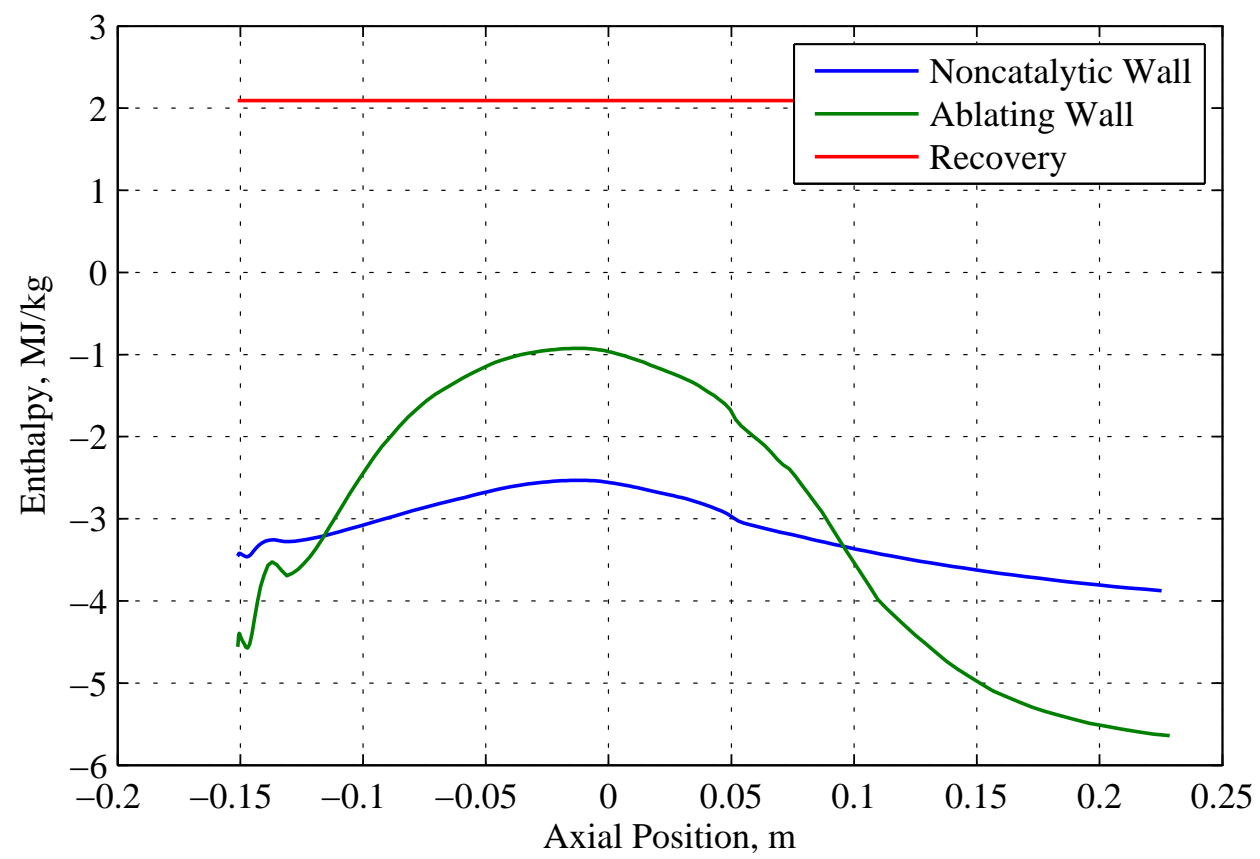

Figure 6. Comparison of noncatalytic wall enthalpy (from the flow solver), ablating wall enthalpy (from the $B^{\prime}$ tables), and recovery enthalpy as a function of axial position for the HIPPO nozzle at $t=0.2 \mathrm{~s}$, as computed with the NCW method.

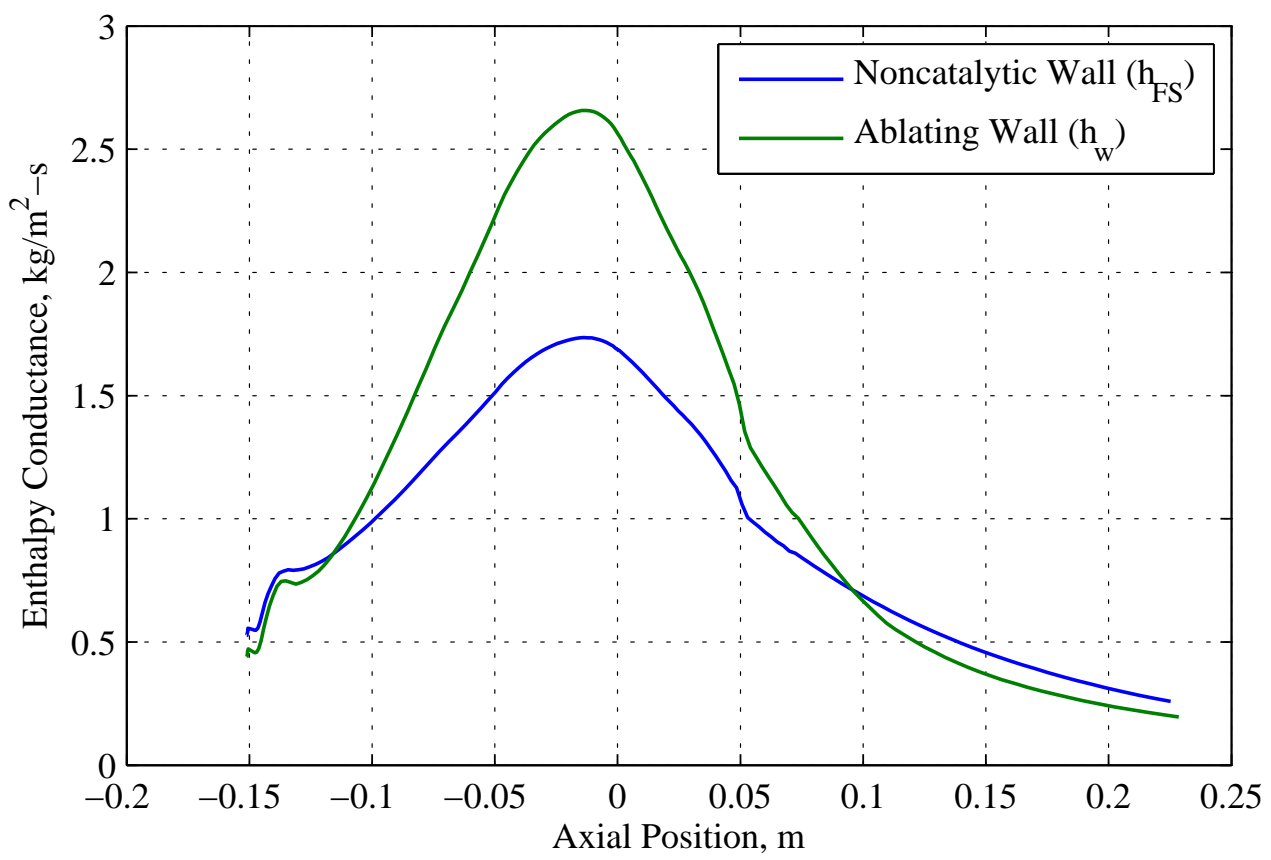

Figure 7. Comparison of enthalpy conductance computed based on the noncatalytic wall and ablating wall enthalpies and the total heat flux (from the NCW method) for the HIPPO nozzle at $t=0.2 \mathrm{~s}$.

11 of 22 
analysis method by inherently capturing the effects of recession (changing geometry), wall temperature, and mass injection (blowing) on the convective heating. (The decoupled analysis approach largely relies on correction factors to account for these effects.) Similar to the decoupled approach, this method uses a transport coefficient assumption to adjust the convection heat heat flux computed by the flow solver to account for the enthalpy at the ablating wall. The NCWC method therefore does not significantly overpredict the ablation response, as is the case for the NCW method. However, this method cannot directly capture the effects of the injection of ablation product species into the flowfield.

The AWT and AWTC methods provide an increased level of modeling fidelity, in that the effects of ablation product species are being partially accounted for (in addition to capturing the effects of recession, wall temperature, and blowing). These two methods can inherently handle the effect that ablation product species diffusion has on the convective heating applied to the ablating wall. However, when performing the surface energy balance (specifically, when computing the heat flux due to pyrolysis gas and char mass fluxes) these approaches still make transport coefficient and unity Lewis number assumptions. This means that these methods will not be able to capture the full effect that species diffusion has on char mass flux (and hence recession rate). The only difference between these two methods is that the AWT method passes raw heat flux from the flow solver to the material response solver, while the AWTC method passes an enthalpy conductance. As will be shown in section III.D, these two methods produce very similar results. However, the AWTC method may permit larger spacing between time points, and hence more affordable conjugate simulations.

The highest level of fidelity is provided by the IEC method. With this method the transport coefficient and unity Lewis number assumptions are not made, and pre-computed $B^{\prime}$ tables are not used. Instead, the actual species diffusion at the ablating wall is used to compute the char mass flux (recession rate). Because the IEC method is directly computing mass diffusion at the ablating wall, it inherently captures the effects of a non-unity Lewis number and of chemical buffering in the boundary layer. This method can therefore fully capture the effect of ablation product species injection into the nozzle flowfield, in addition to the effects of recession, wall temperature, and blowing.

\section{Analysis of the HIPPO Nozzle}

Conjugate ablation analyses are performed for the HIPPO nozzle, 25 a sub-scale $(6.35 \mathrm{~cm}$ diameter throat $)$ space shuttle solid rocket test motor with a carbon-phenolic nozzle. Extensive test data are available for this nozzle, including surface recession and char depth as a function of axial position for eight circumferential stations. This test case was previously the subject of decoupled ablation analyses. 6

\section{III.A. Model}

The geometry of the HIPPO nozzle is illustrated in Fig. 8. No information is available describing the nozzle contour more than about $0.13 \mathrm{~m}$ upstream of the throat. However, based on similar nozzles in the

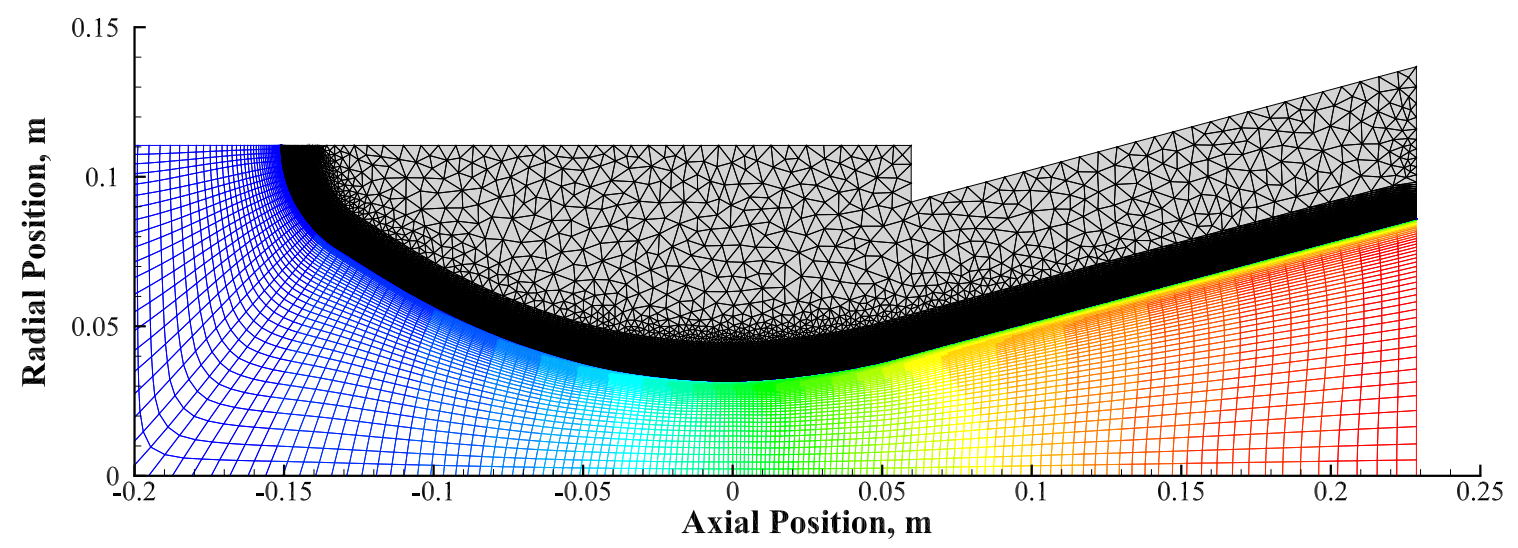

Figure 8. Geometry of the HIPPO nozzle. Shaded region with black mesh lines is the material response solver domain. Mesh in the flow solver domain is also illustrated and colored based on flow velocity. 
literature,, 26 it seems likely that the HIPPO nozzle was submerged, with a nose similar to that assumed and illustrated in Fig. 8.

The mesh for the flowfield is constructed of quadrilateral elements, and has 77 cells in the wall-normal direction and 150 cells in the axial direction, for a total of 11,550 cells. Near wall cell thickness ranges from approximately $6.2 \times 10^{-8} \mathrm{~m}$ at the throat increasing to $2.0 \times 10^{-7} \mathrm{~m}$ at the exit; a stretch ratio of $20 \%$ is used in the wall-normal direction. Wall $Y^{+}$values remain below 0.1 for the length of the nozzle with this mesh. The axial dimension of the cells ranges from approximately $1.27 \times 10^{-3} \mathrm{~m}$ near the throat and nose increasing to $6.8 \times 10^{-3} \mathrm{~m}$ at the exit. Biasing is used in order to obtain a smooth mesh. Mesh parameters are determined from an extensive mesh refinement study, which suggests that this mesh can yield heat flux values with an error of less than $0.5 \%$. The flowfield mesh is illustrated in Fig. 8.

The mesh for the solid domain features a $1.27 \mathrm{~cm}$ thick layer of stacked anisotropic triangular elements adjacent to the ablating boundary. 67 layers of elements are used in this region, with a near-wall thickness of $2.54 \times 10^{-5} \mathrm{~m}$ and a growth rate of 1.05 . This mesh topology is obtained by first generating a structured mesh with quadrilateral cells, then triangulating each cell. It is found that it is important for the diagonal faces dividing the quadrilateral elements to be aligned in the same direction. The axial dimensions of the elements are the same as for the flowfield mesh. Coincident nodes are maintained on the ablating boundary between the flowfield mesh and the material response mesh (though this is not a requirement of the coupled code). Approximately isotropic triangular elements are used to fill the remainder of the domain. Mesh parameters are determined from a mesh refinement study. The final mesh contains 21,926 elements and 11,144 nodes. Material response simulations use a variable time step according to the schedule presented in Table 2; this captures early transient effects while not using an unnecessarily small time step later in the simulation.

The nozzle material is MX4926 carbon-phenolic, and is modeled using properties previously reported.6 $B^{\prime}$ tables are computed using the Chemics ${ }^{27}$ chemical equilibrium program and the CEA 28 thermodynamic database. Gas-phase finite-rate chemistry is modeled using a 20 species, 33 reaction reduced mechanism developed by the authors and presented in a companion paper. 29 Diffusion of gas-phase species is modeled using a constant Lewis number $\mathrm{Le}=0.66\left(\mathrm{Le} \equiv \frac{\mathrm{Pr}}{\mathrm{Sc}}\right)$. Lewis number is obtained from an isentropic expansion calculation performed with the Chemics code. For a chamber pressure of $P_{0}=4.48 \mathrm{MPa}$, Lewis number ranges from $\mathrm{Le}=0.65$ in the chamber to $\mathrm{Le}=0.71$ at the exit, with $\mathrm{Le}=0.66$ occurring at the throat.

\section{III.B. Decoupled Analysis}

While decoupled ablation analyses of the HIPPO nozzle have previously been completed, $\underline{6}$ a new decoupled analysis is performed to provide a more reasonable baseline for comparing to the results from conjugate simulations. This decoupled simulation uses the updated HIPPO geometry that includes the nozzle nose (excluded from the previous study). The general approach taken for this decoupled analysis is to use the same methodology that might be used as the first step in a traditional, decoupled analysis.

The measured pressure trace from the HIPPO motor firing is closely approximated using seven discrete pressures, as illustrated in Fig. 9. Convective boundary conditions for each pressure are computed using the LeMANS flow solver. Wall temperature is set to be a uniform $3000 \mathrm{~K}$, which is about $500 \mathrm{~K}$ less than the chamber temperature. This is consistent with the first iteration of a traditional analysis, and provides a well-defined baseline for comparisons. However, enthalpy conductance (and the resultant thermal response) has been shown to be sensitive to the choice of wall temperature when computing boundary conditions.6 As a result, this decoupled analysis could under-predict the thermal response in the downstream portion of the nozzle, where wall temperatures are expected to be significantly less than the assumed constant value.

Table 2. Time step schedule used for material response simulations.

\begin{tabular}{cc}
\hline Time, s & $\Delta t, \mathrm{~s}$ \\
\hline 0.0 & $1.0 \times 10^{-6}$ \\
$1.0 \times 10^{-5}$ & $1.0 \times 10^{-5}$ \\
$1.0 \times 10^{-4}$ & $1.0 \times 10^{-4}$ \\
0.001 & 0.001 \\
0.01 & 0.01 \\
1.0 & 0.05 \\
\hline
\end{tabular}

13 of 22 


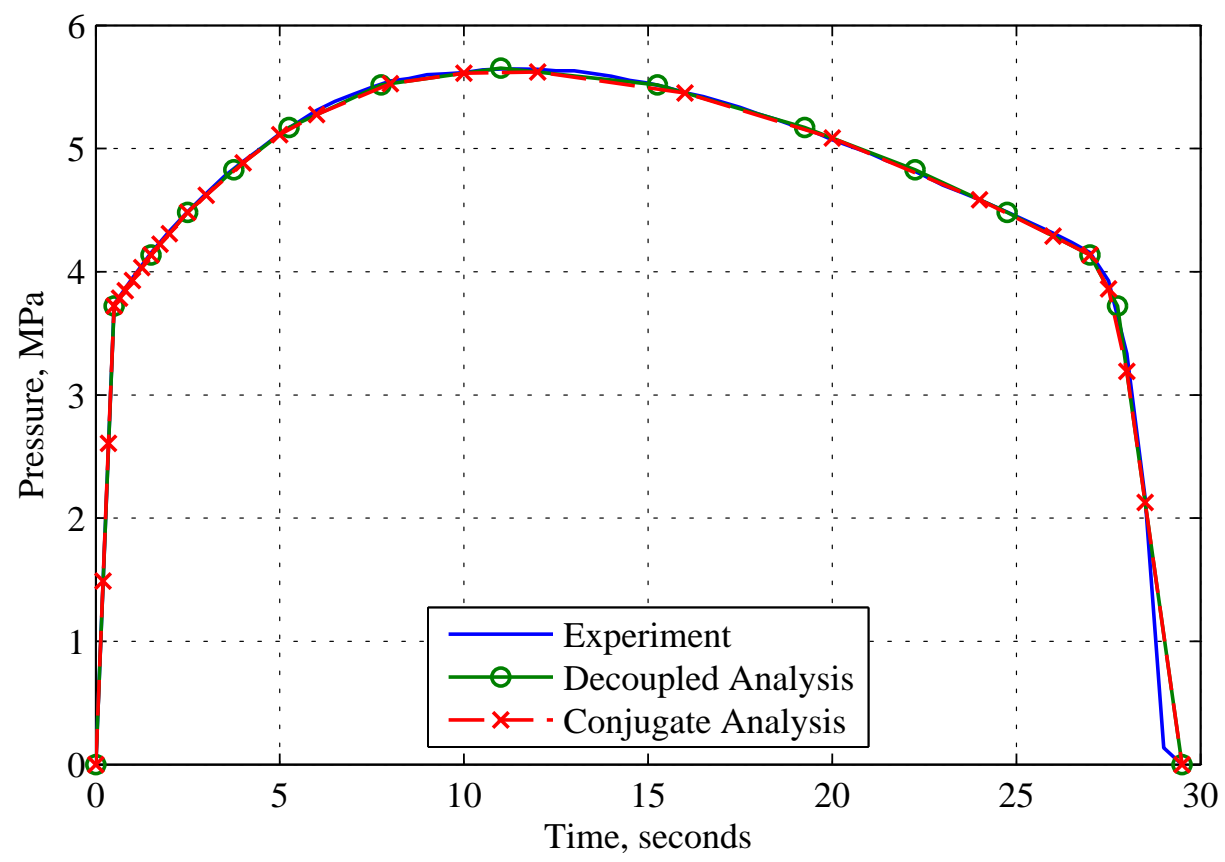

Figure 9. Pressure trace for the HIPPO motor, comparing the experimentally measured pressure trace to the discrete pressure traces used for the decoupled and conjugate analyses.

Recovery enthalpy is taken to be the stagnation enthalpy at each pressure. This is a small departure from the typical approach, where separate simulations using an adiabatic wall boundary condition are performed to obtain a local recovery enthalpy value. The main justification for using the stagnation enthalpy instead of a local recovery enthalpy is to be consistent with the conjugate analyses, where only a stagnation enthalpy value is available. Stagnation enthalpy is greater than the local recovery enthalpy, so this assumption will mitigate to some extent the under-prediction caused by using a constant wall temperature.

Radiation is neglected from all analyses presented in this paper. The main reason for this assumption is to prevent radiation from masking differences between the decoupled and the conjugate simulations, and between the different surface energy balance methods investigated with the conjugate analyses. Additionally, previous work showed that radiation had only a small impact on the thermal response at or downstream of the throat (though radiation made a significant impact upstream of the throat). 6

Surface recession at the end of the motor firing as predicted by this decoupled analysis is compared to experimental measurements in Fig. 10. It is observed that the decoupled analysis over-predicts recession in the region upstream of the nozzle throat. Possible causes for this over-prediction include the unity Lewis number assumption made as part of the surface energy balance, and the inability to capture the effects of recession (modified geometry) on the enthalpy conductance. Downstream of the throat better agreement is achieved. However, this is likely coincidental, and caused by the expected under-prediction of enthalpy conductance in this region.

\section{III.C. Convergence Studies}

A study is performed to investigate the impact that the under-relaxation factor has on the convergence of the conjugate solution, and to identify the optimum value to use. Conjugate simulations are performed for the first time point $(t=0.2 \mathrm{~s})$ for the HIPPO nozzle using the Noncatalytic Wall (NCW) surface energy balance method. Four different under-relaxation values are considered $(\zeta=1.0,0.75,0.5,0.2)$ and are applied to the wall temperature and mass flux. Under-relaxation is not applied to the wall position. Convergence of the conjugate solution at this time point is presented in Fig. 11 and Fig. 12. The RMS average heat flux on the ablating wall is plotted in Fig. 11 as a function of the call to the material response solver. Figure 12 gives the percent RMS difference between the wall temperature predicted by the material response solver and the wall temperature actually used by the flow solver, which is an excellent measure of the convergence of the conjugate solution. 


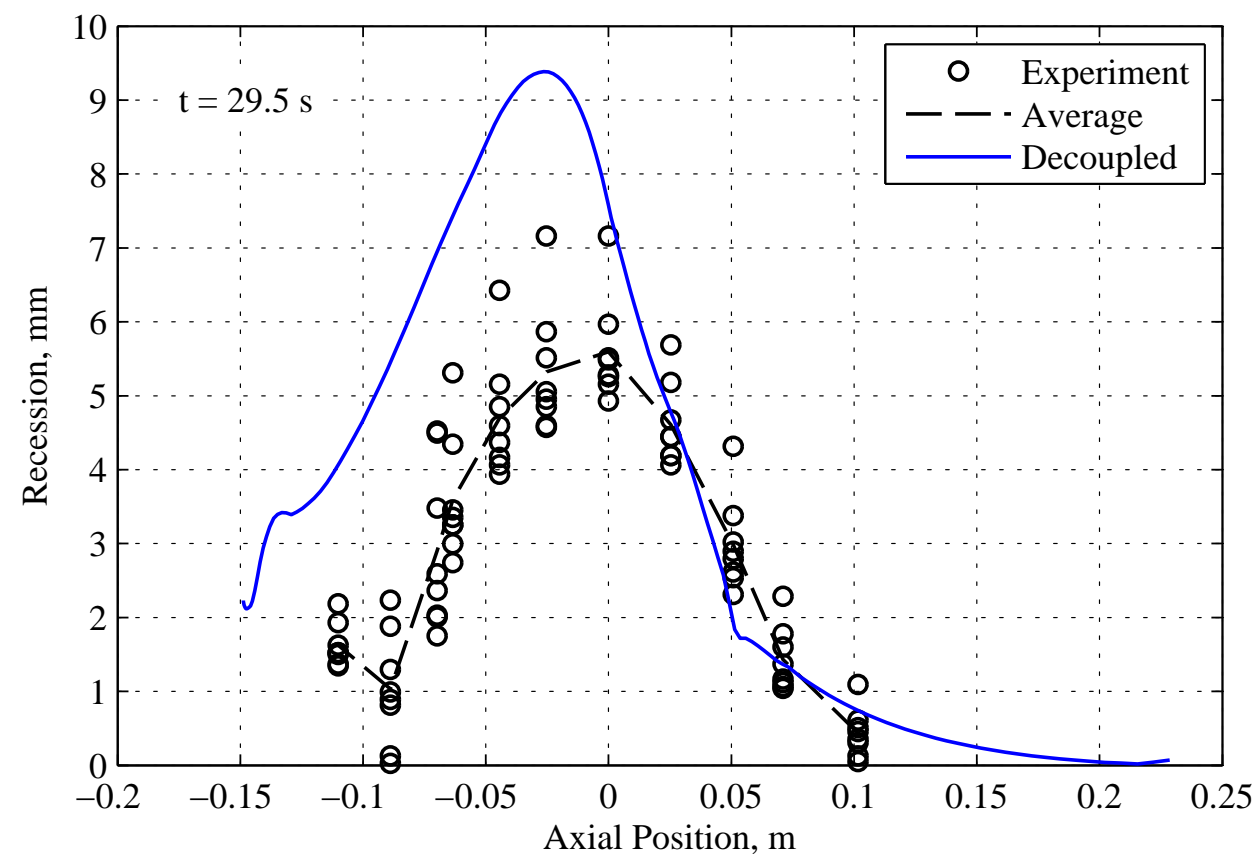

Figure 10. Comparison of predicted and measured surface recession at the end of the HIPPO motor firing as a function of axial position.

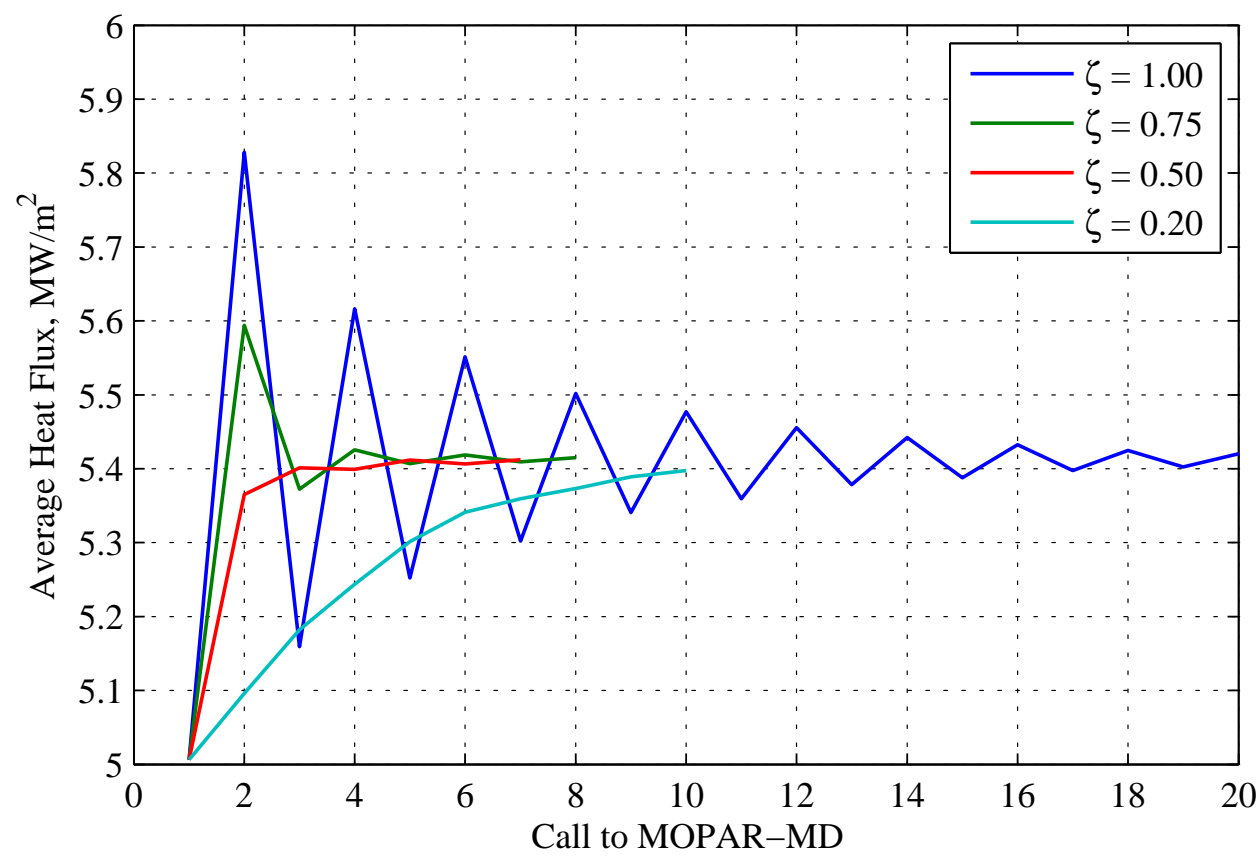

Figure 11. Average heat flux on the ablating boundary as a function of the call to the material response solver and the under-relaxation factor $\zeta$. 


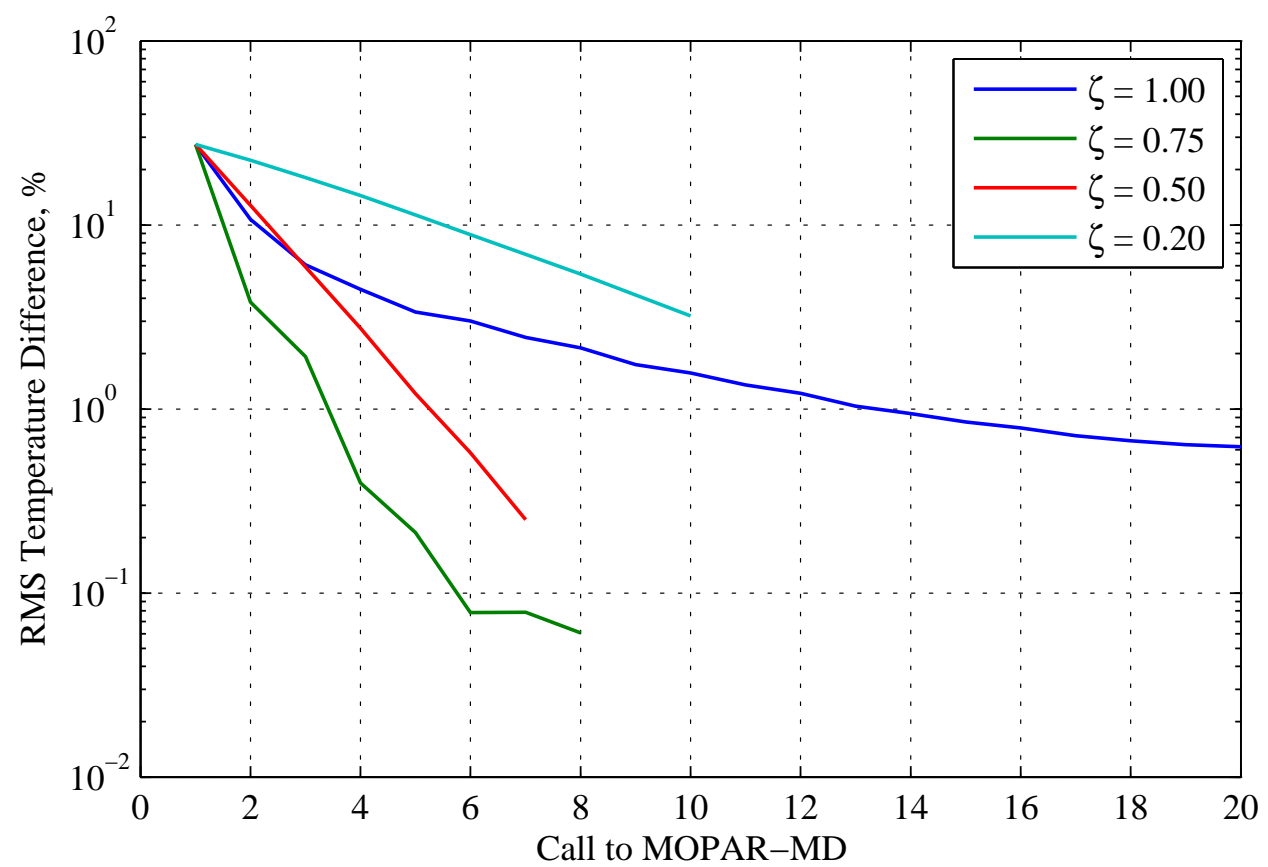

Figure 12. Percent root mean square difference between the wall temperature predicted by the material response solver and the wall temperature used on the flowfield boundary, as a function of the call to the material response solver and the under-relaxation factor $\zeta$.

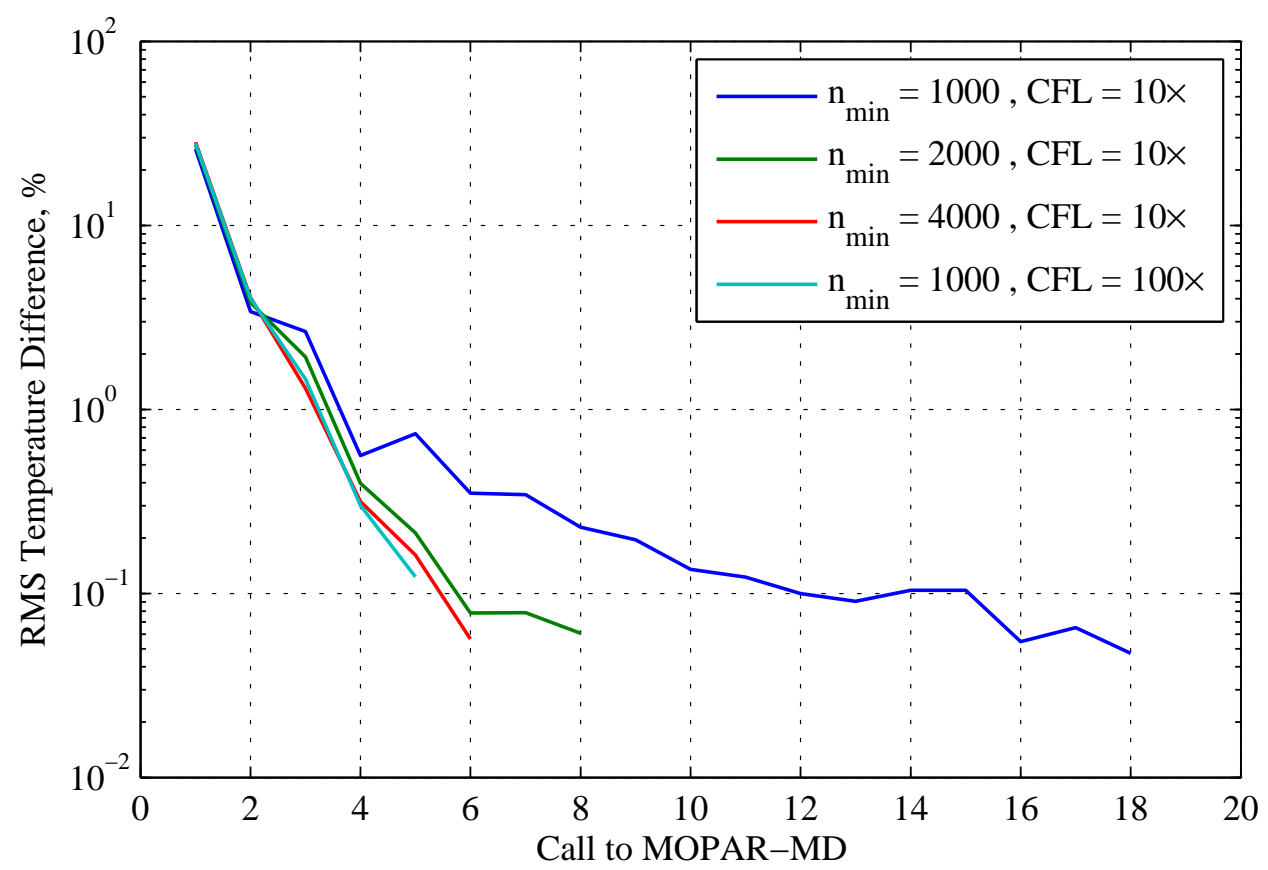

Figure 13. Percent root mean square difference between the wall temperature predicted by the material response solver and the wall temperature used on the flowfield boundary, as a function of call to the material response solver and minimum number of flowfield iterations between calls. 
It can be seen from these plots that using an under-relaxation factor $\zeta=1.0$ (i.e. directly applying new wall values from the material response solver) causes oscillations in the average heat flux, and delays convergence. However, using too low of an under-relaxation factor also hinders convergence of the conjugate solution, which can be most clearly seen by looking at the curve for $\zeta=0.2$ in these plots. The optimum under-relaxation factor for this case appears to be $\zeta=0.75$, which provides very rapid convergence, as can be clearly seen in Fig. 12 .

A second study is conducted to determine the effect that flowfield development has on convergence of the conjugate solution. This is investigated by varying the CFL number ramp and the minimum number of flowfield iterations between calls to the material response solver. Results from this study are presented in Fig. 13. It is discovered that obtaining adequate development of the flowfield between calls to the material response solver is important for obtaining rapid convergence of the conjugate solution. For the simulations using the "10×" CFL ramp (in which the CFL number is ramped from 0.1 to 500 over 5000 iterations), it is found that requiring 2000 flow iterations between material response calls provides better convergence than requiring only 1000 iterations between calls. However, there is negligible added benefit from requiring 4000 flow iterations between calls. Increasing the CFL ramp by an additional factor of ten ("100×"; CFL number is ramped from 1.0 to 500 over 500 iterations) causes the flowfield to develop much more rapidly. As a result, 1000 flow iterations between calls to the material response solver is sufficient for good convergence.

\section{III.D. Conjugate Analyses}

Conjugate flowfield / ablation analyses are performed for the HIPPO nozzle using the five surface energy balance approaches described in section II.E. Variable time point spacing is used, as can be seen in Fig. 9. Time points are closely spaced at the beginning of the conjugate simulations in order to capture the early transients, while the spacing is increased with time, in order to minimize the number of time points analyzed. The interval between time points is selected so that the changes in pressure, heat flux, and temperature at the nozzle throat (as obtained from the decoupled analysis described in section III.B) between time points are approximately equal. Attempts at using larger time point spacing early in the motor firing were not successful. Simulations have been completed only for the first few seconds of the motor firing; simulations for the complete pressure trace are in progress.

Most simulations use an under-relaxation factor of $\zeta=0.5$; higher values can be successfully used for the methods using a noncatalytic wall (NCW, NCWC), but can be unstable for the other methods. For early time points it is sometimes necessary to drop the under-relaxation factor on the species mass fractions to $\zeta=0.25$. It is necessary to use the converged solution obtained with the AWT method as the starting point for the IEC simulation for the first time point. A converged conjugate solution can be obtained after approximately 10 calls to the material response solver for the NCW and NCWC methods, while the other methods require approximately 20 calls.

Surface temperature at the nozzle throat as a function of time as computed by the conjugate simulations is compared in Fig. 14 to results from the decoupled analysis. Surface temperature distribution at $t=2.0 \mathrm{~s}$ is compared in Fig. 15. Upstream of the throat there is fairly good agreement between all simulations. However, at and downstream of the throat all conjugate simulations predict a thermal response more severe than that predicted with the decoupled analysis. Most of this discrepancy can be attributed to the underprediction of the enthalpy conductance for the decoupled analysis (due to the choice of wall temperature when computing the convective heating). This under-prediction would be most significant at and downstream of the nozzle throat. The NCW method significantly over-predicts the thermal response due to the reasons discussed in section II.E.6. Since this method is found to be inappropriate for conjugate ablation analyses of rocket nozzles, simulations with this method are discontinued after $t=1.0 \mathrm{~s}$. The AWT, AWTC, and IEC methods are in very close agreement with each other, and produce thermal responses most similar to the decoupled analysis results. The NCWC method predicts a thermal response that is approximately $2 \%$ higher than that obtained with the AWT, AWTC, and IEC methods.

Surface recession at the nozzle throat is compared as a function of time in Fig. 16. The NCW method grossly over-predicts recession due to the over-prediction of both heat flux and enthalpy conductance, as discussed in section II.E.6. Surface recession distribution at $t=2.0 \mathrm{~s}$ is compared in Fig. 17. The NCWC method provides more reasonable recession values, but still predicts recession values that are substantially greater than those obtained with the AWT and AWTC methods. This difference is related to the fact that the NCWC method predicts enthalpy conductance values that are higher than those obtained with the AWT and AWTC methods. Surface recession values as predicted by the AWT and AWTC methods are nearly

17 of 22

American Institute of Aeronautics and Astronautics 


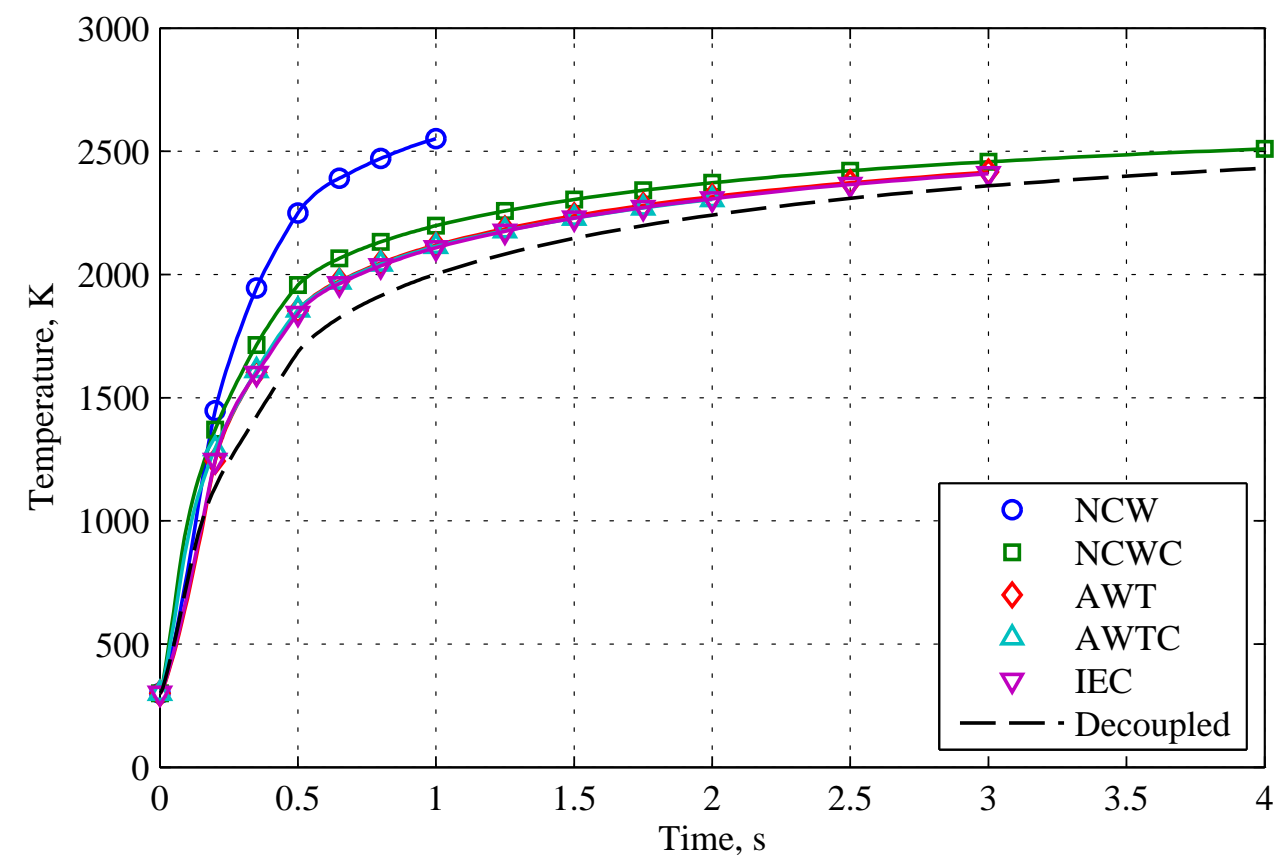

Figure 14. Surface temperature at the throat of the HIPPO nozzle as a function of time, as computed by the decoupled analysis and by conjugate simulations using the different surface energy balance approaches.

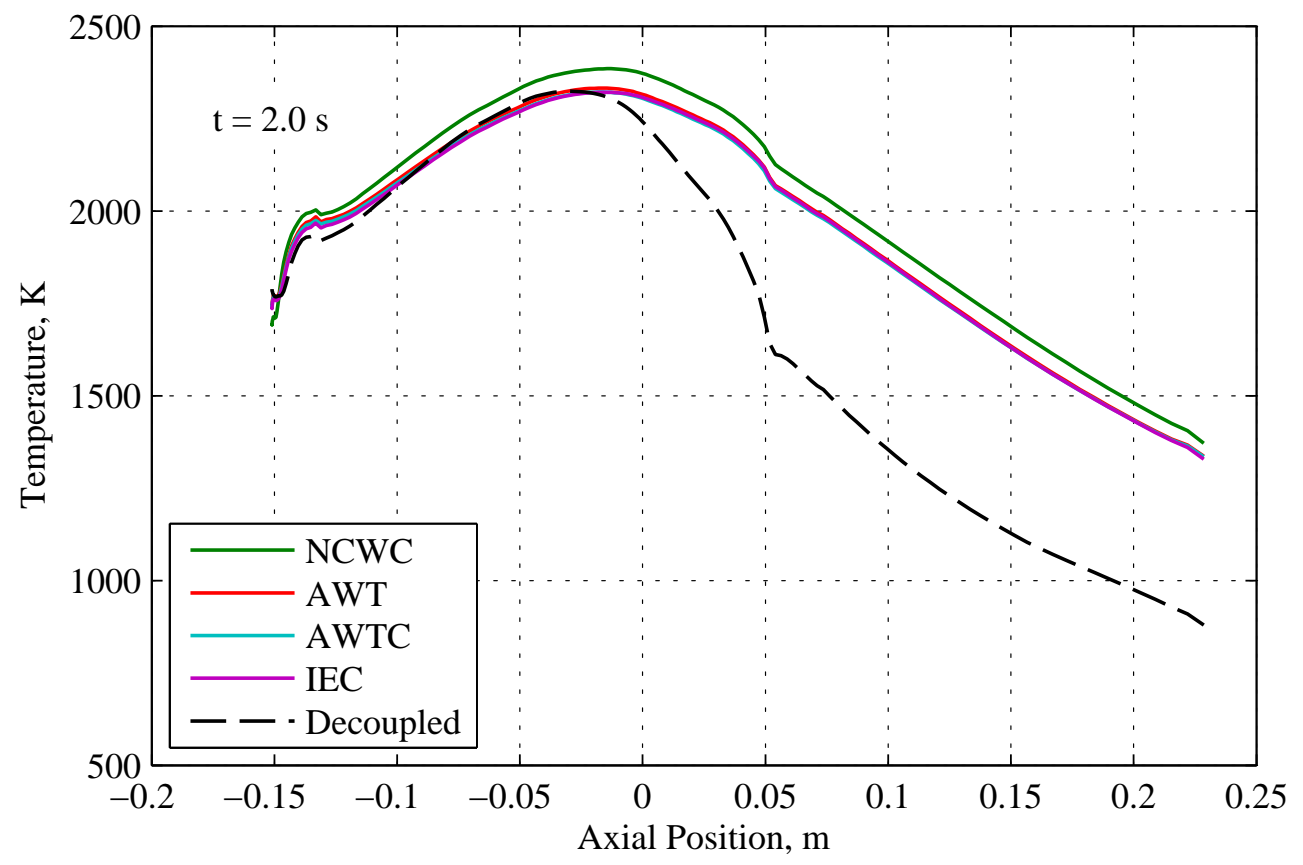

Figure 15. Surface temperature distribution within the HIPPO nozzle at $t=2.0 \mathrm{~s}$, as computed by the decoupled analysis and by conjugate simulations using the different surface energy balance approaches. 


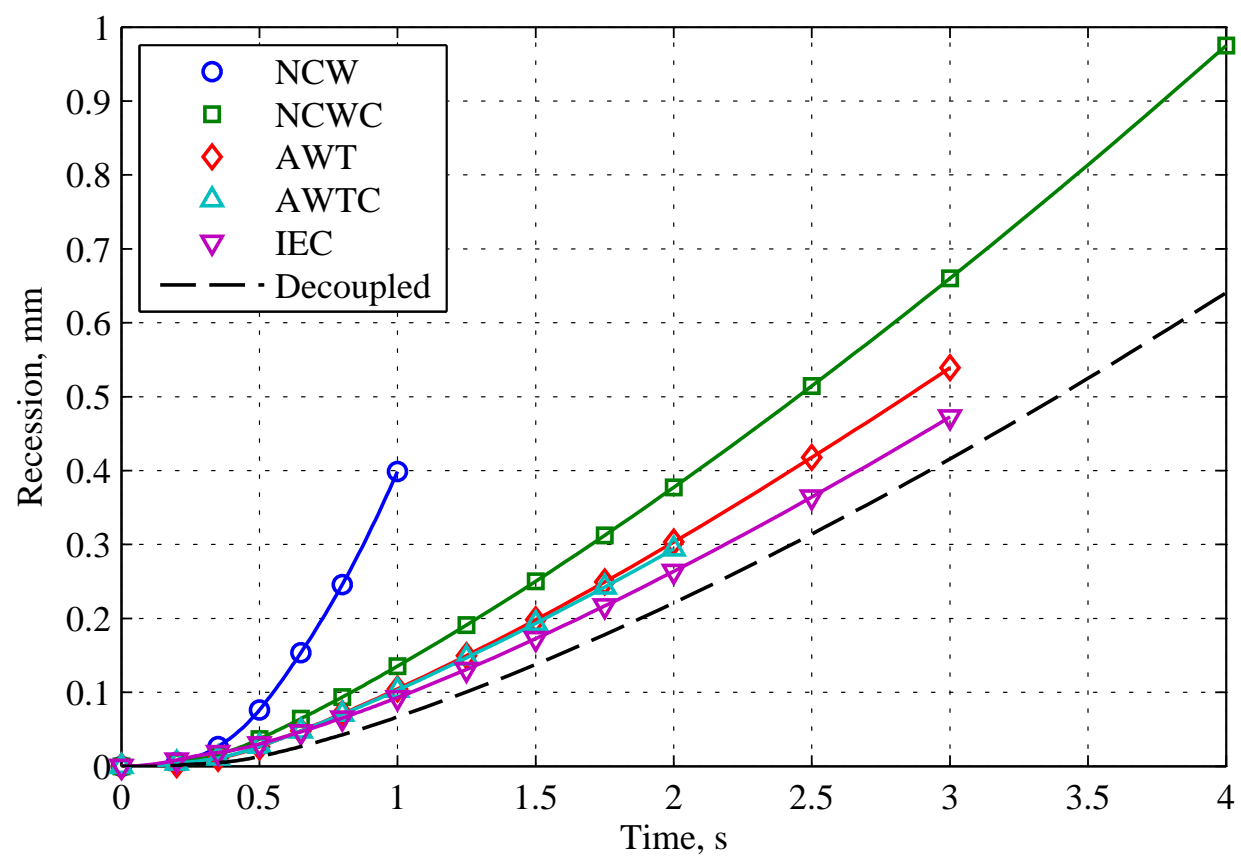

Figure 16. Surface recession at the throat of the HIPPO nozzle as a function of time, as computed by the decoupled analysis and by conjugate simulations using the different surface energy balance approaches.

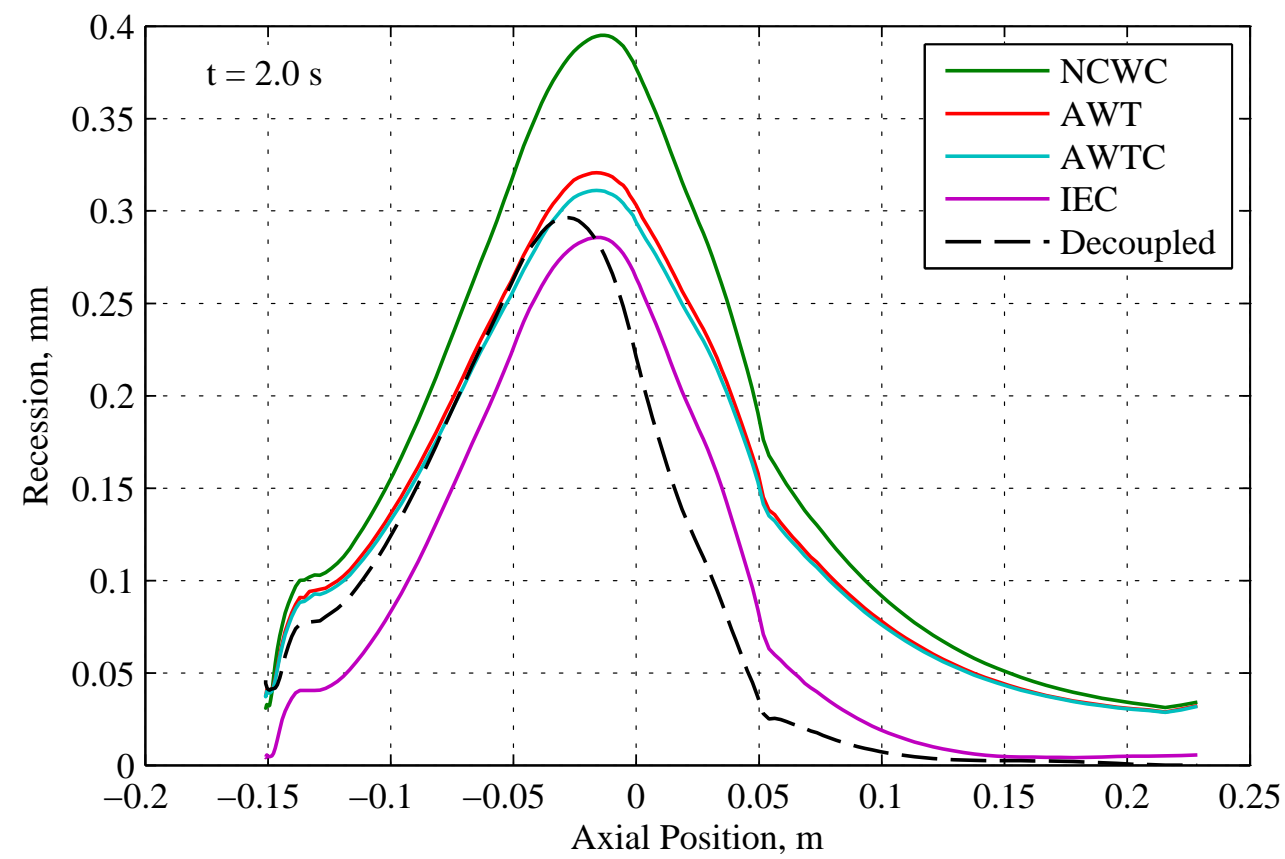

Figure 17. Surface recession distribution within the HIPPO nozzle at $t=2.0 \mathrm{~s}$, as computed by the decoupled analysis and by conjugate simulations using the different surface energy balance approaches. 
identical, and are in good agreement with the decoupled analysis results upstream of the throat. However, recession is still significantly over-predicted at and downstream of the throat. Of the conjugate simulation approaches, the IEC method predicts the lowest surface recession; the recession distribution is very similar to that obtained with the AWT and AWTC methods, but shifted downwards. Upstream of the throat the IEC method actually predicts less recession than that obtained with the decoupled analysis. At and shortly downstream of the throat the IEC method over-predicts recession relative to the decoupled analysis, but good agreement is obtained between the two methods near the nozzle exit.

There are several reasons why the surface recession as predicted by the IEC method is lower than that predicted with the other conjugate approaches. All the other methods model mass diffusion at the ablating wall using a transport coefficient assumption, while the IEC method directly models the diffusion of species to and from the ablating wall. Diffusion for the IEC method is based on a Lewis number of Le $=0.66$, while the other methods are based on an assumption of unity Lewis number (equal heat and mass transport coefficients). All things being equal, a lower Lewis number should give less mass diffusion, and thus lower recession. Finally, since the IEC method is directly computing mass diffusion, it inherently captures the effects of the buildup of ablation product species in the boundary layer due to upstream mass injection. This chemical buffering of the boundary layer would tend to suppress ablation at downstream locations. The methods relying on a transport coefficient assumption cannot account for this chemical buffering.

Proper comparison of the results produced with the IEC method to experimental data is dependent upon completion of a conjugate simulation for the full pressure trace, an endeavor which is currently in progress. However, preliminary comparisons can be made by extrapolating the trends observed in Fig. 17, and comparing to the experimental results in Fig. 10. Peak recession occurs closer to the nozzle throat with the IEC method than with the decoupled analysis; in this regard the IEC method agrees better with the experimental results than the decoupled analysis. Upstream of the throat the IEC method predicts less recession than the decoupled analysis; this would bring the IEC results in closer agreement with the experimental results than the decoupled analysis. However, downstream of the throat the IEC method is predicting greater recession than the decoupled analysis, which could lead to worse agreement with experimental measurements in this region.

\section{Conclusion}

A methodology enabling fully-coupled, conjugate, two-dimensional simulations of ablation of pyrolyzing materials in rocket nozzle applications has been presented. A rocket motor firing pressure trace is divided into a number of discrete time points, with a steady-state flowfield solution obtained at each point. Wall boundary conditions for the flow solver come from transient material response analyses. Convergence of the conjugate solution is obtained in an iterative process, with the material response solver being called multiple times for each time point. Under-relaxation of values passed from the material response solver to the flow solver helps with the convergence of the conjugate solution. The ideal under-relaxation factor appears to be $\zeta \approx 0.5$; larger values can be unstable, while lower values can delay convergence. Convergence studies also showed that it is important to obtain adequate development of the flowfield between calls to the material response solver.

Five different treatments of the surface energy balance were presented, with increasing levels of fidelity. The NCW method was found to be unsuitable for rocket nozzle applications, even though it has been used in the literature for modeling external thermal protection systems. This method over-predicts both heat flux and enthalpy conductance, leading to significant over-predictions of the ablation response. The NCWC method corrects some of these issues, but still uses a noncatalytic wall boundary condition for the flow solver. This method therefore cannot directly capture the effects of the injection of ablation product species into the flowfield, but does account for recession, wall temperature, and blowing. The AWT and AWTC methods can account for the effect that ablation product species have on the heat flux at the ablating wall, but still rely on transport coefficient and unity Lewis number assumptions and pre-computed $B^{\prime}$ tables for the computation of char mass flux and recession. These two methods give very similar results, but the AWTC method should permit greater spacing between time points and therefore more affordable simulations.

The highest fidelity surface energy balance approach is the IEC method, which abandons the the transport coefficient and unity Lewis number assumptions and therefore does not require the use of pre-computed $B^{\prime}$ tables. Char mass flux is computed based on the actual species diffusion at the ablating wall, and can therefore inherently capture the effects of a non-unity Lewis number and of chemical buffering (the buildup 
of ablation product species in the boundary layer due to upstream mass injection) in the boundary layer.

The thermal and ablation response of the HIPPO nozzle test case was predicted using a decoupled analysis technique as well as the five conjugate methods presented here. The decoupled analysis over-predicts recession upstream of the throat, while good agreement with experimental data is obtained downstream of the throat. However, this good agreement is believed to be coincidental and the result of a known under-prediction of the convective heating environment in this region for the decoupled analysis. Conjugate simulations have only been completed for the first few seconds of the motor firing. Surface temperature as predicted with the AWT, AWTC, and IEC methods is almost identical, while the NCWC values are approximately $2 \%$ higher. The NCW method greatly over-predicts surface temperature and recession. The NCWC method predicts more recession throughout the nozzle than was predicted with the decoupled method, while the AWT and AWTC methods give good agreement in the region upstream of the throat. The IEC method predicts the lowest recession, and in fact predicts less recession in the region upstream of the throat than obtained from the decoupled analysis. Extrapolations suggest that the IEC method should provide better agreement with experimental recession data than the other conjugate approaches or the decoupled analysis method. Future work will focus on extending the conjugate simulations for the full duration of the motor firing.

By solving the ablation problem in a fully conjugate manner, many of the simplifying assumptions that must be made in traditional, decoupled ablation modeling approaches are avoided. The strong interactions and interdependencies that exist between the reacting flowfield and the ablating material are captured rigorously. This places the analysis more strongly upon first principles, and should improve the accuracy of the resulting predictions.

\section{Acknowledgments}

Funding for this work was provided by the NAWCWD Fellowship Program, the SMART Scholarship, and NAWCWD IAR 5097. Computational resources were provided in part by the Department of Defense High Performance Computing Modernization Office.

\section{References}

\footnotetext{
${ }^{1}$ Bartz, D. R., "A Simple Equation for Rapid Estimation of Rocket Nozzle Convective Heat Transfer Coefficients," Jet Propulsion, Vol. 37, No. 1, January 1957, pp. 49-51.

${ }^{2}$ Aerotherm Corporation, Huntsville, AL, User's Manual, Nozzle Aero Thermochemistry (NAT) Computer Code, Version 2.0, February 1994.

${ }^{3}$ Acurex Corporation, Mountain View, CA, User's Manual, Non-Proprietary, Aerotherm Charring Material Thermal Response and Ablation Program, CMA87S, November 1987.

${ }^{4}$ Kuntz, D. W., Hassan, B., and Potter, D. L., "Predictions of Ablating Hypersonic Vehicles Using an Iterative Coupled Fluid/Thermal Approach," Journal of Thermophysics and Heat Transfer, Vol. 15, No. 2, Apr 2001, pp. 129-139.

${ }^{5}$ Johnston, C. O., Gnoffo, P. A., and Mazaheri, A., "Study of Ablation-Flowfield Coupling Relevant to the Orion Heat Shield," Journal of Thermophysics and Heat Transfer, Vol. 26, No. 2, April-June 2012, pp. 213-221.

${ }^{6}$ Cross, P. G. and Boyd, I. D., "Two-Dimensional Modeling of Ablation and Pyrolysis with Application to Rocket Nozzles," Journal of Spacecraft and Rockets, Vol. 54, No. 1, January 2017, pp. 212-224.

${ }^{7}$ Bianchi, D., Turchi, A., Nasuti, F., and Onofri, M., "Chemical Erosion of Carbon-Phenolic Rocket Nozzles with FiniteRate Surface Chemistry," Journal of Propulsion and Power, June 2013, pp. 1220-1230.

${ }^{8}$ Bianchi, D. and Nasuti, F., "Carbon-Carbon Nozzle Erosion and Shape-Change Effects in Full-Scale Solid-Rocket Motors," Journal of Propulsion and Power, Vol. 28, No. 4, July 2012, pp. 820-830.

$\checkmark{ }^{9}$ Bianchi, D., Nasuti, F., Onofri, M., and Martelli, E., "Thermochemical Erosion Analysis for Graphite/Carbon-Carbon Rocket Nozzles," Journal of Propulsion and Power, Vol. 27, No. 1, Jan 2011, pp. 197-205.

${ }^{10}$ Bianchi, D., Nasuti, F., and Martelli, E., "Navier-Stokes Simulations of Hypersonic Flows with Coupled Graphite Ablation," Journal of Spacecraft and Rockets, Vol. 47, No. 4, Jul 2010, pp. 554-562.

${ }^{11}$ Bianchi, D., Nasuti, F., and Martelli, E., "Coupled Analysis of Flow and Surface Ablation in Carbon-Carbon Rocket Nozzles," Journal of Spacecraft and Rockets, Vol. 46, No. 3, May 2009, pp. 492-500.

12 Thakre, P. and Yang, V., "Chemical Erosion of Carbon-Carbon/Graphite Nozzles in Solid-Propellant Rocket Motors," Journal of Propulsion and Power, Vol. 24, No. 4, Jul 2008, pp. 822-833.

13 Martin, A. and Boyd, I. D., "Strongly Coupled Computation of Material Response and Nonequilibrium Flow for Hypersonic Ablation," Journal of Spacecraft and Rockets, Vol. 25, 2015, pp. 89-104.

${ }^{14}$ Alkandry, H., Boyd, I. D., and Martin, A., "Coupled Flow Field Simulations of Charring Ablators with Nonequilibrium Surface Chemistry," 44th AIAA Thermophysics Conference, June 2013, AIAA 2013-2634.

${ }^{15}$ Wiebenga, J. and Boyd, I., "Computation of Multi-Dimensional Material Response Coupled to Hypersonic Flow," 43rd AIAA Thermophysics Conference, Jun 2012, AIAA 2012-2873.
} 
${ }^{16}$ Thompson, R. and Gnoffo, P., "Implementation of a Blowing Boundary Condition in the LAURA Code," 46th AIAA Aerospace Sciences Meeting and Exhibit, Jan 2008, AIAA 2008-1243.

$\checkmark{ }^{17}$ Olynick, D., Chen, Y.-K., and Tauber, M. E., "Aerothermodynamics of the Stardust Sample Return Capsule," Journal of Spacecraft and Rockets, Vol. 36, No. 3, May 1999, pp. 442-462.

${ }^{18}$ Gnoffo, P. A. and Johnston, C. O., "A Boundary Condition Relaxation Algorithm for Strongly Coupled, Ablating Flows including Shape Change," 42nd AIAA Thermophysics Conference, Honolulu, HI, 27-30 June 2011, AIAA $2011-3760$.

${ }^{19}$ Martin, A., Scalabrin, L. C., and Boyd, I. D., "High performance modeling of atmospheric re-entry vehicles," Journal of Physics: Conference Series, Vol. 341, February 2012, pp. 012002.

${ }^{20}$ Scalabrin, L. C., Numerical Simulation of Weakly Ionized Hypersonic Flow Over Reentry Capsules, Ph.D. thesis, University of Michigan, Ann Arbor, MI, 2007.

${ }^{21}$ Wiebenga, J. E., High-Fidelity Material Response Modeling as Part of an Aerothermoelastic Framework for Hypersonic Flows, Ph.D. thesis, University of Michigan, Ann Arbor, MI, 2014.

${ }^{22}$ Blackwell, B. and Howard, M., "An Element Potential Based Chemical Equilibrium Solver for Gas/Surface Thermochemistry," 50th AIAA Aerospace Sciences Meeting including the New Horizons Forum and Aerospace Exposition, January 2012, AIAA 2012-0815.

${ }^{23}$ Milos, F. and Chen, Y.-K., "Ablation, Thermal Response, and Chemistry Program for Analysis of Thermal Protection Systems," 10th AIAA/ASME Joint Thermophysics and Heat Transfer Conference, June 2010, AIAA 2010-4663.

${ }^{24}$ Scoggins, J. B. and Magin, T. E., "Development of Mutation++: Multicomponent Thermodynamic and Transport Properties for Ionized Plasmas written in C++," 11th AIAA/ASME Joint Thermophysics and Heat Transfer Conference, June 2014, AIAA 2014-2966.

${ }^{25}$ Arnold, J., Dodson, J., and Laub, B., "Subscale Solid Motor Nozzle Tests - Phase IV and Nozzle Materials Screening and Thermal Characterization - Phase V," NASA CR 161254, NASA, June 1979.

${ }^{26}$ Kearney, W. J. and Ellis, R. A., "Improved Performance Solid Rocket Nozzles of Advanced and Low-Cost Carbon/Carbon Materials," 1979 JANNAF Propulsion Meeting, Anaheim, CA, March 1979, pp. 115-132.

27Ewing, M. E. and Isaac, D. A., "Mathematical Modeling of Multiphase Chemical Equilibrium," Journal of Thermophysics and Heat Transfer, Vol. 29, No. 3, July 2015, pp. 551-562.

${ }^{28} \mathrm{McBride}, \mathrm{B}$. J. and Gordon, S., "Computer Program for Calculation of Complex Chemical Equilibrium Compositions and Applications. Part 2: User's Manual and Program Description," NASA RP-1311-P2, NASA, June 1996.

${ }^{29}$ Cross, P. G. and Boyd, I. D., "Reduced Reaction Mechanism for Rocket Nozzle Ablation Simulations," 4 th AIAA Thermophysics Conference, June 2017. 